\title{
Existence and exponentially stability of anti-periodic solutions for neutral BAM neural networks with time-varying delays in the leakage terms
}

\author{
Changjin $\mathrm{Xu}^{\mathrm{a}, *}$, Peiluan $\mathrm{Li}^{\mathrm{b}}$ \\ a Guizhou Key Laboratory of Economics System Simulation Guizhou, University of Finance and Economics, Guiyang 550004, China. \\ ${ }^{b}$ School of Mathematics and Statistics, Henan University of Science and Technology, Luoyang 471023, China.
}

Communicated by R. Saadati

\begin{abstract}
This paper is concerned with the existence and exponential stability of anti-periodic solutions of a neutral BAM neural network with time-varying delays in the leakage terms. Using some analysis skills and Lyapunov method, a series of sufficient conditions for the existence and exponential stability of anti-periodic solutions to the neutral BAM neural networks with time-varying delays in the leakage terms are presented. Our results are new and complement some previously known ones. (C)2016 All rights reserved.
\end{abstract}

Keywords: Neutral BAM neural network, anti-periodic solution, exponential stability, time-varying delay, leakage term.

2010 MSC: 34C25, 34K13, 34K25.

\section{Introduction}

In the last three decades, considerable attention has been paid to BAM neural networks as well as various generalizations for their essential applications in classification, pattern recognition, optimization, signal and image processing, parallel computation, associative memory and nonlinear optimization problems and so on [1, 2, 7, 9, 11, 13, 15, 17, 21, 25, 28, 29, 30, 33, 34, 37, 38, 39, 40, 41, 42. Recently, Li and Li [16] studied the following neutral delay BAM neural networks with time-varying delays in leakage terms

\footnotetext{
${ }^{*}$ Corresponding author

Email addresses: xcj403@126.com (Changjin Xu), lpllpl_lpl@163.com (Peiluan Li)
} 


$$
\left\{\begin{aligned}
\frac{d x_{i}(t)}{d t}= & -a_{i}(t) x_{i}\left(t-\alpha_{i}(t)\right)+\sum_{j=1}^{m} a_{j i}(t) f_{j}\left(y_{j}\left(t-\tau_{j i}(t)\right)\right) \\
& +\sum_{j=1}^{m} p_{j i}(t) g_{j}\left(y_{j}^{\prime}\left(t-\sigma_{j i}(t)\right)\right)+I_{i}(t), \\
\frac{d y_{j}(t)}{d t}= & -b_{j}(t) y_{j}\left(t-\beta_{j}(t)\right)+\sum_{i=1}^{n} b_{i j}(t) h_{i}\left(x_{i}\left(t-\xi_{i j}(t)\right)\right) \\
& +\sum_{i=1}^{n} q_{i j}(t) k_{i}\left(x_{i}^{\prime}\left(t-\varsigma_{i j}(t)\right)\right)+J_{i}(t),
\end{aligned}\right.
$$

where $i=1,2, \cdots, n ; j=1,2, \cdots, m, t \in R, n, m$ are the number of neurons in layers, $x_{i}(t)$ and $y_{i}(t)$ denote the activations of the $i$-th neuron and the $j$ th neuron at time $t ; a_{i}$ and $b_{j}$ represent the rate with which the $i$ th neuron and $j$ th neuron will reset their potential to the resting state in isolation when they are disconnected from the network and the external inputs at time $t ; f_{j}, g_{j}, h_{j}$ and $k_{j}$ are the input-output functions (the activation functions); $\alpha_{i}, \beta_{j}, \tau_{j i}, \sigma_{j i}, \xi_{i j}$ and $\varsigma_{i j}$ are transmission delays at time $t ; a_{j i}$ and $p_{j i}$ are elements of feedback templates at time $t$ and $b_{i j}, q_{i j}$ are elements of feed-forward templates at tiem $t ; I_{i}, J_{j}$ denote biases of the $i$ th neuron and the $j$ th neuron at time $t, i=1,2, \ldots, n, j=1,2, \ldots, m$. By using the exponential dichotomy of linear differential equations, fixed point theorems and constructing a Lyapunov functional, Li and Li [16] obtained some sufficient conditions to guarantee the existence and exponential stability of almost periodic solution for model (1.1).

We know that the existence and stability of anti-periodic solutions play a key role in characterizing the behavior of nonlinear differential equations [3, 4, 10, 14, 18, 19, 20, 22, 23, 24, 26, 27, 31, 32, 35, 36. For example, the signal transmission process of neural networks can often be described as an anti-periodic process. In recent years, the anti-periodic problems of neural networks have been investigated by numerous scholars [3, 4, 10, 18, 19, 20, 22, 23, 24, 26, 31, 35. Considering that a typical time delay called Leakage (or "forgetting") delay may exist in the negative feedback term of the neural networks system (these terms are variously known as forgetting or leakage terms), some authors discussed the anti-periodic solution of neural networks with delays in the leakage terms (see [5, 6, 8, 12, 14, 20, 24, 27, 36]). Very recently, another type of time-delays, namely, neutral-type time-delays which always appears in the study of automatic control, population dynamics and vibrating masses attached to an elastic bar, etc., has recently drawn considerable attention [16].

To the best of our knowledge, up to now, there are very few papers that focus on the existence and stability of anti-periodic solution to neutral BAM neural networks with time-varying delays in the leakage terms. Thus, it is important and necessary to investigate the existence and stability of anti-periodic solution for neutral BAM neural networks with time-varying delays in the leakage terms.

The purpose of this paper is to focus on the existence and exponential stability of anti-periodic solution of system (1.1). By the fundamental solution matrix, Lyapunov function and constructing fundamental function sequences based on the solution of networks, we establish a series of sufficient conditions of existence and global exponential stability of anti-periodic solutions of the neutral BAM neural network with timevarying delays in the leakage terms. Our results not only can be applied directly to many concrete examples of neural networks, but also extend, to a certain extent, some previously known ones. In addition, an example is given to illustrate the feasibility and effectiveness of our main results. Our results are a good complement of $\mathrm{Li}$ and $\mathrm{Li}[16$.

The organization of the rest of this paper is as follows. In Section 2, we give some notations and preliminary results. In Section 3, we present some sufficient conditions for the existence and global exponential stability of anti-periodic solution of the neutral BAM neural network with time-varying delays in the leakage terms. In Section 4, an example is given to illustrate the feasibility and effectiveness of our main theoretical findings in previous sections. 


\section{Preliminary results}

In this section, we shall present some notations and introduce some lemmas which are used in the following sections.

For any vector $V=\left(v_{1}, v_{2}, \ldots, v_{n}\right)^{T}$ and matrix $D=\left(d_{i j}\right)_{n \times n}$, we define the norm as

$$
\|v\|=\left(\sum_{i=1}^{n} v_{i}^{2}\right)^{\frac{1}{2}},\|D\|=\left(\sum_{i=1}^{n} d_{i j}^{2}\right)^{\frac{1}{2}}
$$

respectively. Let

$$
\begin{aligned}
\tau= & \left\{\sup _{t \in R} \max _{1 \leq i \leq n} \alpha_{i}(t), \sup _{t \in R} \max _{1 \leq i \leq n, 1 \leq j \leq m} \tau_{j i}(t), \sup _{t \in R} \max _{1 \leq i \leq n, 1 \leq j \leq m} \sigma_{j i}(t),\right. \\
& \left.\sup _{t \in R} \max _{1 \leq j \leq m} \beta_{j}(t), \sup _{t \in R} \max _{1 \leq i \leq n, 1 \leq j \leq m} \xi_{i j}(t), \sup _{t \in R} \max _{1 \leq i \leq n, 1 \leq j \leq m} \varsigma_{i j}(t)\right\}, \\
\varphi(s)= & \left(\varphi_{1}(s), \varphi_{2}(s), \cdots, \varphi_{n}(s)\right)^{T}, \psi(s)=\left(\psi_{1}(s), \psi_{2}(s), \cdots, \psi_{m}(s)\right)^{T},
\end{aligned}
$$

where $\varphi_{i}(s) \in C([-\tau, 0], R), \psi_{j}(s) \in C([-\tau, 0], R), i=1,2, \cdots, n, j=1,2, \cdots, m$. We define

$$
\|\varphi\|=\sup _{-\tau \leq s \leq 0}\left(\sum_{i=1}^{n}\left|\varphi_{i}(s)\right|^{2}\right)^{\frac{1}{2}},\|\psi\|=\sup _{-\tau \leq s \leq 0}\left(\sum_{j=1}^{m}\left|\psi_{j}(s)\right|^{2}\right)^{\frac{1}{2}} .
$$

We assume that system (1.1) always satisfies the following initial conditions:

$$
\left\{\begin{array}{l}
x_{i 0}=\varphi_{i}(s), s \in[-\tau, 0] \\
y_{j 0}=\psi_{j}(s), s \in[-\tau, 0]
\end{array}\right.
$$

Let $x(t)=\left(x_{1}(t), x_{2}(t), \cdots, x_{n}(t)\right)^{T}, y(t)=\left(y_{1}(t), y_{2}(t), \cdots, y_{m}(t)\right)^{T}$ be the solution of system (1.1) with initial conditions 2.1]. We say the solution $x(t)=\left(x_{1}(t), x_{2}(t), \cdots, x_{n}(t)\right)^{T}, y(t)=\left(y_{1}(t), y_{2}(t), \cdots, y_{m}(t)\right)^{T}$ is $T$-anti-periodic on $R^{n+m}$ if $x_{i}(t+T)=-x_{i}(t), y_{j}(t+T)=-y_{j}(t)$ for all $t \in R$ and $i=1,2, \ldots, n, j=$ $1,2, \ldots, m$, where $T$ is a positive constant.

Throughout this paper, for $i=1,2, \cdots, n, j=1,2, \cdots, m$, it will be assumed that there exist constants such that

$$
\begin{aligned}
& a_{i}^{+}=\sup _{t \in R} a_{i}(t), b_{j}^{+}=\sup _{t \in R} b_{j}(t), a_{j i}^{+}=\sup _{t \in R} a_{j i}(t), p_{j i}^{+}=\sup _{t \in R} p_{j i}(t), I_{i}^{+}=\sup _{t \in R} I_{i}(t), \\
& b_{i j}^{+}=\sup _{t \in R} b_{i j}(t), q_{i j}^{+}=\sup _{t \in R} q_{i j}(t), a_{i}^{-}=\inf _{t \in R} a_{i}(t), b_{i}^{-}=\inf _{t \in R} b_{i}(t), J_{i}^{+}=\sup _{t \in R} J_{j}(t) .
\end{aligned}
$$

We also assume that the following conditions hold.

(H1) For $i=1,2, \cdots, n, j=1,2, \cdots, m$, there exist constants $L_{j f}>0, L_{j g}>0, L_{i h}>0, L_{i k}>0, M_{j}^{f}>$ $0, M_{j}^{g}>0, M_{i}^{h}>0$ and $M_{i}^{k}>0$ such that

$$
\left\{\begin{array}{l}
\left|f_{j}(u)-f_{j}(v)\right| \leq L_{j f}|u-v|,\left|f_{j}(u)\right|<M_{j}^{f} \\
\left|g_{j}(u)-g_{j}(v)\right| \leq L_{j g}|u-v|,\left|g_{j}(u)\right|<M_{j}^{g} \\
\left|h_{i}(u)-h_{i}(v)\right| \leq L_{i h}|u-v|,\left|h_{i}(u)\right|<M_{i}^{h} \\
\left|k_{i}(u)-k_{i}(v)\right| \leq L_{i k}|u-v|,\left|k_{i}(u)\right|<M_{i}^{k}
\end{array}\right.
$$

for all $u, v \in R$.

(H2) For all $t, u \in R$ and $i=1,2, \cdots, n, j=1,2, \cdots, m$,

$$
\left\{\begin{array}{l}
a_{j i}(t+T) f_{j}(u)=-a_{j i}(t) f_{j}(-u), p_{j i}(t+T) g_{j}(u)=-p_{j i}(t) g_{j}(-u), \\
b_{i j}(t+T) h_{i}(u)=-b_{i j}(t) h_{i}(-u), q_{i j}(t+T) k_{i}(u)=-q_{i j}(t) k_{i}(-u), \\
\alpha_{i}(t+T)=\alpha_{i}(t), \tau_{j i}(t+T)=\tau_{j i}(t), \sigma_{j i}(t+T)=\sigma_{j i}(t), I_{i}(t+T)=-I_{i}(t), \\
\beta_{j}(t+T)=\beta_{i}(t), \xi_{i j}(t+T)=\xi_{i j}(t), \varsigma_{i j}(t+T)=\varsigma_{i j}(t), J_{i}(t+T)=-J_{i}(t),
\end{array}\right.
$$


where $T$ is a positive constant.

(H3) The following inequality holds.

$$
\frac{\sqrt{2}}{\alpha}\left(\left(a_{i}^{+}\right)^{2} \alpha_{i}^{+}+\left(b_{j}^{+}\right)^{2} \beta_{j}^{+}\right)<1,
$$

where $\alpha=\min _{1 \leq i \leq n, 1 \leq j \leq m}\left\{a_{i}^{-}, b_{j}^{-}\right\}, i=1,2, \ldots, n, j=1,2, \ldots, m$.

Definition 2.1. The solution $\left(x^{*}(t), y^{*}(t)\right)^{T}$ of system 1.1 is said to be globally exponentially stable if there exist constants $\beta>0$ and $M>1$ such that

$$
\sum_{i=1}^{n}\left|x_{i}(t)-x_{i}^{*}(t)\right|^{2}+\sum_{j=1}^{m}\left|y_{j}(t)-y_{j}^{*}(t)\right|^{2} \leq M e^{-\beta t}\left[\left\|\varphi-\varphi^{*}\right\|^{2}+\left\|\psi-\psi^{*}\right\|^{2}\right]
$$

for each solution $(x(t), y(t))^{T}$ of system 1.1.

Next, we present three important lemmas which are necessary for proving our main results in Section 3 .

\section{Lemma 2.2. Let}

$$
A=\left(\begin{array}{cc}
-a_{i}^{-} & 0 \\
0 & -b_{j}^{-}
\end{array}\right), \alpha=\min _{1 \leq i \leq n}\left\{a_{i}^{-}, b_{j}^{-}\right\}
$$

then we have

$$
\|\exp A t\| \leq \sqrt{2} e^{-\alpha t}
$$

for all $t \geq 0$.

Proof. Since

$$
A=\left(\begin{array}{cc}
-a_{i}^{-} & 0 \\
0 & -b_{j}^{-}
\end{array}\right)
$$

it follows that

$$
\exp A t=\left(\begin{array}{cc}
e^{-a_{i}^{-} t} & 0 \\
0 & e^{-b_{j}^{-} t}
\end{array}\right)
$$

By the definition of matrix norm, we get

$$
\|\exp A t\|=\left(e^{-2 a_{i}^{-} t}+e^{-2 b_{j}^{-} t}\right)^{\frac{1}{2}} \leq \sqrt{2} e^{-\alpha t} .
$$

Lemma 2.3. Assume that

$$
(\mathrm{H} 4)\left\{\begin{array}{l}
-2 a_{i}^{-}+a_{i}^{+} \alpha_{i}^{+}\left(a_{i}^{+}+\sum_{j=1}^{m} a_{j i}^{+} L_{j f}^{2 \xi_{j}}+\sum_{j=1}^{m} p_{j i}^{+} L_{j g}^{2 \gamma_{j}}\right) \\
+\sum_{j=1}^{m} a_{j i}^{+} L_{j f}^{2 \xi_{j}}+\sum_{j=1}^{m} p_{j i}^{+} L_{j g}^{2 \gamma_{j}}+\sum_{j=1}^{m} b_{j}^{+} \beta_{j} b_{i j}^{+} L_{j h}^{2\left(1-\varepsilon_{i}\right)} \\
+\sum_{j=1}^{m} b_{i j}^{+} L_{j h}^{2\left(1-\varepsilon_{i}\right)}+\sum_{j=1}^{m} q_{i j}^{+} L_{j h}^{2 \iota_{i}}+\sum_{j=1}^{m} b_{j}^{+} \beta_{j}^{+} q_{i j}^{+} L_{j h}^{2\left(1-\iota_{i}\right)}<0, \\
-2 b_{j}^{-}+b_{j}^{+} \beta_{j}^{+}\left(b_{j}^{+}+\sum_{i=1}^{n} b_{i j}^{+} L_{i h}^{2 \varepsilon_{i}}+\sum_{i=1}^{n} q_{i j}^{+} L_{i h}^{2 \iota_{i}}\right) \\
+\sum_{i=1}^{n} b_{i j}^{+} L_{i h}^{2 \varepsilon_{i}}+\sum_{i=1}^{n} a_{i}^{+} \alpha_{i}^{+} a_{j i}^{+} L_{j f}^{2\left(1-\varepsilon_{j}\right)}+\sum_{i=1}^{n} a_{j i}^{+} L_{j f}^{2\left(1-\xi_{j}\right)} \\
+\sum_{i=1}^{n} a_{i}^{+} \alpha_{i}^{+} p_{j i}^{+} L_{j g}^{2\left(1-\gamma_{j}\right)}+\sum_{i=1}^{n} p_{j i}^{+} L_{j g}^{2\left(1-\gamma_{j}\right)}+\sum_{i=1}^{n} a_{j i}^{+} L_{j f}^{2\left(1-\xi_{j}\right)}<0,
\end{array}\right.
$$


where $0 \leq \xi_{j}, \gamma_{j}, \varepsilon_{i}, \varepsilon_{j}, \iota_{i} \leq 1(i=1,2, \cdots, n, j=1,2, \cdots, m)$ are any constants. Then there exists $\beta>0$ such that

$$
\begin{aligned}
& \beta-2 a_{i}^{-}+a_{i}^{+} \alpha_{i}^{+}\left(a_{i}^{+}+\sum_{j=1}^{m} a_{j i}^{+} L_{j f}^{2 \xi_{j}}+\sum_{j=1}^{m} p_{j i}^{+} L_{j g}^{2 \gamma_{j}}\right) \\
& +\sum_{j=1}^{m} a_{j i}^{+} L_{j f}^{2 \xi_{j}}+\sum_{j=1}^{m} p_{j i}^{+} L_{j g}^{2 \gamma_{j}}+\sum_{j=1}^{m} b_{j}^{+} \beta_{j} b_{i j}^{+} L_{j h}^{2\left(1-\varepsilon_{i}\right)} e^{\beta \tau} \\
& +\sum_{j=1}^{m} b_{i j}^{+} L_{j h}^{2\left(1-\varepsilon_{i}\right)} e^{\beta \tau}+\sum_{j=1}^{m} q_{i j}^{+} L_{j h}^{2 \iota_{i}} e^{\beta \tau}+\sum_{j=1}^{m} b_{j}^{+} \beta_{j}^{+} q_{i j}^{+} L_{j h}^{2\left(1-\iota_{i}\right)} e^{\beta \tau} \leq 0, \\
& \beta-2 b_{j}^{-}+b_{j}^{+} \beta_{j}^{+}\left(b_{j}^{+}+\sum_{i=1}^{n} b_{i j}^{+} L_{i h}^{2 \varepsilon_{i}}+\sum_{i=1}^{n} q_{i j}^{+} L_{i h}^{2 \iota_{i}}\right) \\
& +\sum_{i=1}^{n} b_{i j}^{+} L_{i h}^{2 \varepsilon_{i}}+\sum_{i=1}^{n} a_{i}^{+} \alpha_{i}^{+} a_{j i}^{+} L_{j f}^{2\left(1-\varepsilon_{j}\right)} e^{\beta \tau}+\sum_{i=1}^{n} a_{j i}^{+} L_{j f}^{2\left(1-\xi_{j}\right)} e^{\beta \tau} \\
& +\sum_{i=1}^{n} a_{i}^{+} \alpha_{i}^{+} p_{j i}^{+} L_{j g}^{2\left(1-\gamma_{j}\right)} e^{\beta \tau}+\sum_{i=1}^{n} p_{j i}^{+} L_{j g}^{2\left(1-\gamma_{j}\right)} e^{\beta \tau}+\sum_{i=1}^{n} a_{j i}^{+} L_{j f}^{2\left(1-\xi_{j}\right)} e^{\beta \tau} \leq 0 .
\end{aligned}
$$

Proof. Let

$$
\begin{aligned}
\varrho_{1 i}(\beta)= & \beta-2 a_{i}^{-}+a_{i}^{+} \alpha_{i}^{+}\left(a_{i}^{+}+\sum_{j=1}^{m} a_{j i}^{+} L_{j f}^{2 \xi_{j}}+\sum_{j=1}^{m} p_{j i}^{+} L_{j g}^{2 \gamma_{j}}\right) \\
& +\sum_{j=1}^{m} a_{j i}^{+} L_{j f}^{2 \xi_{j}}+\sum_{j=1}^{m} p_{j i}^{+} L_{j g}^{2 \gamma_{j}}+\sum_{j=1}^{m} b_{j}^{+} \beta_{j} b_{i j}^{+} L_{j h}^{2\left(1-\varepsilon_{i}\right)} e^{\beta \tau} \\
& +\sum_{j=1}^{m} b_{i j}^{+} L_{j h}^{2\left(1-\varepsilon_{i}\right)} e^{\beta \tau}+\sum_{j=1}^{m} q_{i j}^{+} L_{j h}^{2 \iota_{i}} e^{\beta \tau}+\sum_{j=1}^{m} b_{j}^{+} \beta_{j}^{+} q_{i j}^{+} L_{j h}^{2\left(1-\iota_{i}\right)} e^{\beta \tau}, \\
\varrho_{2 j}(\beta)= & \beta-2 b_{j}^{-}+b_{j}^{+} \beta_{j}^{+}\left(b_{j}^{+}+\sum_{i=1}^{n} b_{i j}^{+} L_{i h}^{2 \varepsilon_{i}}+\sum_{i=1}^{n} q_{i j}^{+} L_{i h}^{2 \iota_{i}}\right) \\
& +\sum_{i=1}^{n} b_{i j}^{+} L_{i h}^{2 \varepsilon_{i}}+\sum_{i=1}^{n} a_{i}^{+} \alpha_{i}^{+} a_{j i}^{+} L_{j f}^{2\left(1-\varepsilon_{j}\right)} e^{\beta \tau}+\sum_{i=1}^{n} a_{j i}^{+} L_{j f}^{2\left(1-\xi_{j}\right)} e^{\beta \tau} \\
& +\sum_{i=1}^{n} a_{i}^{+} \alpha_{i}^{+} p_{j i}^{+} L_{j g}^{2\left(1-\gamma_{j}\right)} e^{\beta \tau}+\sum_{i=1}^{n} p_{j i}^{+} L_{j g}^{2\left(1-\gamma_{j}\right)} e^{\beta \tau}+\sum_{i=1}^{n} a_{j i}^{+} L_{j f}^{2\left(1-\xi_{j}\right)} e^{\beta \tau} .
\end{aligned}
$$

Obviously, $\varrho_{1 i}(\beta), \varrho_{2 j}(\beta)(j=1,2, \cdots, n ; j=1,2, \cdots, m)$ is continuously differential function. We can easily check that

$$
\left\{\begin{array}{l}
\frac{d \varrho_{1 i}(\beta)}{d \beta}>0, \lim _{\beta \rightarrow+\infty} \varrho_{1 i}(\beta)=+\infty, \varrho_{1 i}(0)<0, \\
\frac{d \varrho_{2 j}(\beta)}{d \beta}>0, \lim _{\beta \rightarrow+\infty} \varrho_{2 j}(\beta)=+\infty, \varrho_{2 j}(0)<0 .
\end{array}\right.
$$

By using the intermediate value theorem, there exist constants $\beta_{i}>0(i=1,2, \cdots, n), \beta_{j}^{*}>0(j=$ $1,2, \cdots, m)$ such that

$$
\varrho_{1 i}\left(\beta_{i}\right)=0, i=1,2, \cdots, n, \varrho_{2 j}\left(\beta_{j}^{*}\right)=0, j=1,2, \cdots, m \text {. }
$$


Let $\beta_{0}=\min \left\{\beta_{1}, \beta_{2}, \cdots, \beta_{n}, \beta_{1}^{*}, \beta_{2}^{*}, \cdots, \beta_{m}^{*}\right\}$, then it follows that $\beta_{0}>0$ and

$$
\varrho_{1 i}\left(\beta_{0}\right) \leq 0, i=1,2, \cdots, n, \varrho_{2 j}\left(\beta_{0}\right) \leq 0, j=1,2, \cdots, m .
$$

This completes the proof of Lemma 2.3 .

Lemma 2.4. Assume that (H1) and (H3) are satisfied. Then for any solution $\left(x_{1}(t), x_{2}(t), \cdots, x_{n}(t)\right.$, $\left.y_{1}, y_{2}, \cdots, y_{m}\right)^{T}$ of system (1.1) there exists a constant

$$
\begin{aligned}
\sigma^{*}= & {\left[1-\frac{\sqrt{2}}{\alpha}\left(\left(a_{i}^{+}\right)^{2} \alpha_{i}^{+}+\left(b_{j}^{+}\right)^{2} \beta_{j}^{+}\right)\right]^{-1}\left\{\sqrt{2}\left(\|\varphi\|^{2}+\|\psi\|^{2}\right)+\frac{\sqrt{2}}{\alpha}\right.} \\
& \times\left[a_{i}^{+} \alpha_{i}^{+}\left(\sum_{j=1}^{m} a_{j i}^{+} M_{j}^{f}++\sum_{j=1}^{m} p_{j i}^{+} M_{j}^{g}+I_{i}^{+}\right)\right. \\
& +b_{j}^{+} \beta_{j}^{+}\left(\sum_{i=1}^{n} b_{i j}^{+} M_{i}^{h}+\sum_{i=1}^{n} q_{i j}^{+} M_{i}^{k}+J_{j}^{+}\right) \\
& \left.\left.+\sum_{j=1}^{m} a_{j i}^{+} M_{j}^{f}+\sum_{j=1}^{m} p_{j i}^{+} M_{j}^{g}+I_{i}^{+}+\sum_{i=1}^{n} b_{i j}^{+} M_{i}^{h}+\sum_{i=1}^{n} q_{i j}^{+} M_{i}^{k}+J_{j}^{+}\right]\right\}
\end{aligned}
$$

such that

$$
\left|x_{i}(t)\right| \leq \sigma^{*},\left|y_{j}(t)\right| \leq \sigma^{*}, i=1,2, \cdots, n, j=1,2, \cdots, m
$$

for all $t>0$.

Proof. From (1.1), we have

$$
\left\{\begin{aligned}
\frac{d x_{i}(t)}{d t}= & -a_{i}(t) x_{i}(t)+a_{i}(t)\left[x_{i}(t)-x_{i}\left(t-\alpha_{i}(t)\right)\right]+\sum_{j=1}^{m} a_{j i}(t) f_{j}\left(y_{j}\left(t-\tau_{j i}(t)\right)\right) \\
& +\sum_{j=1}^{m} p_{j i}(t) g_{j}\left(y_{j}^{\prime}\left(t-\sigma_{j i}(t)\right)\right)+I_{i}(t), \\
\frac{d y_{j}(t)}{d t}= & -b_{j}(t) y_{j}(t)+b_{j}(t)\left[y_{j}(t)-y_{j}\left(t-\beta_{j}(t)\right)\right]+\sum_{i=1}^{n} b_{i j}(t) h_{i}\left(x_{i}\left(t-\xi_{i j}(t)\right)\right) \\
& +\sum_{i=1}^{n} q_{i j}(t) k_{i}\left(x_{i}^{\prime}\left(t-\varsigma_{i j}(t)\right)\right)+J_{i}(t) .
\end{aligned}\right.
$$

Then we have

$$
\left\{\begin{aligned}
\frac{d x_{i}(t)}{d t}= & -a_{i}(t) x_{i}(t)+a_{i}(t) \int_{t-\alpha_{i}(t)}^{t} x_{i}^{\prime}(s) d s+\sum_{j=1}^{m} a_{j i}(t) f_{j}\left(y_{j}\left(t-\tau_{j i}(t)\right)\right) \\
& +\sum_{j=1}^{m} p_{j i}(t) g_{j}\left(y_{j}^{\prime}\left(t-\sigma_{j i}(t)\right)\right)+I_{i}(t), \\
\frac{d y_{j}(t)}{d t}= & -b_{j}(t) y_{j}(t)+b_{j}(t) \int_{t-\beta_{j}(t)}^{t} y_{j}^{\prime}(s) d s+\sum_{i=1}^{n} b_{i j}(t) h_{i}\left(x_{i}\left(t-\xi_{i j}(t)\right)\right) \\
& +\sum_{i=1}^{n} q_{i j}(t) k_{i}\left(x_{i}^{\prime}\left(t-\varsigma_{i j}(t)\right)\right)+J_{i}(t) .
\end{aligned}\right.
$$


Thus

$$
\left\{\begin{aligned}
\frac{d x_{i}(t)}{d t}= & -a_{i}(t) x_{i}(t)+a_{i}(t) \int_{t-\alpha_{i}(t)}^{t}\left[-a_{i}(s) x_{i}\left(s-\alpha_{i}(s)\right)\right. \\
& \left.+\sum_{j=1}^{m} a_{j i}(s) f_{j}\left(y_{j}\left(s-\tau_{j i}(s)\right)\right)+\sum_{j=1}^{m} p_{j i}(t) g_{j}\left(y_{j}^{\prime}\left(s-\sigma_{j i}(s)\right)\right)+I_{i}(s)\right] d s \\
& +\sum_{j=1}^{m} a_{j i}(t) f_{j}\left(y_{j}\left(t-\tau_{j i}(t)\right)\right)+\sum_{j=1}^{m} p_{j i}(t) g_{j}\left(y_{j}^{\prime}\left(t-\sigma_{j i}(t)\right)\right)+I_{i}(t), \\
\frac{d y_{j}(t)}{d t}= & -b_{j}(t) y_{j}(t)+b_{j}(t) \int_{t-\beta_{j}(t)}^{t}\left[-b_{j}(s) y_{j}\left(s-\beta_{j}(s)\right)\right. \\
& \left.+\sum_{i=1}^{n} b_{i j}(s) h_{i}\left(x_{i}\left(s-\xi_{i j}(s)\right)\right)+\sum_{i=1}^{n} q_{i j}(s) k_{i}\left(x_{i}^{\prime}\left(s-\varsigma_{i j}(s)\right)\right)+J_{i}(s)\right] d s \\
& +\sum_{i=1}^{n} b_{i j}(t) h_{i}\left(x_{i}\left(t-\xi_{i j}(t)\right)\right)+\sum_{i=1}^{n} q_{i j}(t) k_{i}\left(x_{i}^{\prime}\left(t-\varsigma_{i j}(t)\right)\right)+J_{i}(t) .
\end{aligned}\right.
$$

Let

$$
\begin{aligned}
& z_{i j}(t)=\left(\begin{array}{l}
x_{i}(t) \\
y_{j}(t)
\end{array}\right), A=\left(\begin{array}{cc}
-a_{i}^{-} & 0 \\
0 & -b_{j}^{-}
\end{array}\right), \\
& f\left(x_{i}(t), y_{j}(t)\right)=\left(\begin{array}{l}
f_{1}\left(x_{i}(t), y_{j}(t)\right. \\
f_{2}\left(x_{i}(t), y_{j}(t)\right.
\end{array}\right), I_{i j}(t)=\left(\begin{array}{c}
I_{i}(t) \\
J_{j}(t)
\end{array}\right),
\end{aligned}
$$

where

$$
\begin{aligned}
f_{1}\left(x_{i}(t), y_{j}(t)\right)= & a_{i}(t) \int_{t-\alpha_{i}(t)}^{t}\left[-a_{i}(s) x_{i}\left(s-\alpha_{i}(s)\right)+\sum_{j=1}^{m} a_{j i}(s) f_{j}\left(y_{j}\left(s-\tau_{j i}(s)\right)\right)\right. \\
& \left.+\sum_{j=1}^{m} p_{j i}(t) g_{j}\left(y_{j}^{\prime}\left(s-\sigma_{j i}(s)\right)\right)+I_{i}(s)\right] d s \\
& +\sum_{j=1}^{m} a_{j i}(t) f_{j}\left(y_{j}\left(t-\tau_{j i}(t)\right)\right)+\sum_{j=1}^{m} p_{j i}(t) g_{j}\left(y_{j}^{\prime}\left(t-\sigma_{j i}(t)\right)\right), \\
f_{2}\left(x_{i}(t), y_{j}(t)\right)= & b_{j}(t) \int_{t-\beta_{j}(t)}^{t}\left[-b_{j}(s) y_{j}\left(s-\beta_{j}(s)\right)+\sum_{i=1}^{n} b_{i j}(s) h_{i}\left(x_{i}\left(s-\xi_{i j}(s)\right)\right)\right. \\
& \left.+\sum_{i=1}^{n} q_{i j}(s) k_{i}\left(x_{i}^{\prime}\left(s-\varsigma_{i j}(s)\right)\right)+J_{i}(s)\right] d s \\
& +\sum_{i=1}^{n} b_{i j}(t) h_{i}\left(x_{i}\left(t-\xi_{i j}(t)\right)\right)+\sum_{i=1}^{n} q_{i j}(t) k_{i}\left(x_{i}^{\prime}\left(t-\varsigma_{i j}(t)\right)\right),
\end{aligned}
$$

then system 1.1 can be written as the following equivalent form

$$
z_{i j}^{\prime}(t) \leq A z_{i j}(t)+f\left(x_{i}(t), y_{j}(t)\right)+I_{i j}(t) .
$$

Solving the inequality 2.5 , we have 


$$
z_{i j}(t) \leq e^{A t} z_{i j}(0)+\int_{0}^{t} e^{A(t-s)}\left[f\left(x_{i}(s), y_{j}(s)\right)+I_{i j}(t)\right] d s .
$$

It follows from Lemma 2.2 that

$$
\begin{aligned}
\left\|z_{i j}(t)\right\| \leq & \sqrt{2} e^{-\alpha t}\left\|z_{i j}(0)\right\|+\sqrt{2} \int_{0}^{t} e^{-\alpha(t-s)}\left[\left\|f\left(x_{i}(s), y_{j}(s)\right)\right\|+\left|I_{i j}(s)\right|\right] d s \\
\leq & \sqrt{2}\left(\|\varphi\|^{2}+\|\psi\|^{2}\right)+\frac{\sqrt{2}}{\alpha}\left(1-e^{-\alpha t}\right) \\
& {\left[a_{i}^{+} \alpha_{i}^{+}\left(a_{i}^{+}\left|x_{i}\left(t-\alpha_{i}(t)\right)\right|+\sum_{j=1}^{m} a_{j i}^{+} M_{j}^{f}++\sum_{j=1}^{m} p_{j i}^{+} M_{j}^{g}+I_{i}^{+}\right)\right.} \\
& +b_{j}^{+} \beta_{j}^{+}\left(b_{j}^{+}\left|y_{j}\left(t-\beta_{j}(t)\right)\right|+\sum_{i=1}^{n} b_{i j}^{+} M_{i}^{h}+\sum_{i=1}^{n} q_{i j}^{+} M_{i}^{k}+J_{j}^{+}\right) \\
& \left.+\sum_{j=1}^{m} a_{j i}^{+} M_{j}^{f}+\sum_{j=1}^{m} p_{j i}^{+} M_{j}^{g}+I_{i}^{+}+\sum_{i=1}^{n} b_{i j}^{+} M_{i}^{h}+\sum_{i=1}^{n} q_{i j}^{+} M_{i}^{k}+J_{j}^{+}\right] \\
\leq & \sqrt{2}\left(\|\varphi\|^{2}+\|\psi\|^{2}\right)+\frac{\sqrt{2}}{\alpha} \\
& \times\left[a_{i}^{+} \alpha_{i}^{+}\left(a_{i}^{+}\left\|z_{i j}\right\|+\sum_{j=1}^{m} a_{j i}^{+} M_{j}^{f}++\sum_{j=1}^{m} p_{j i}^{+} M_{j}^{g}+I_{i}^{+}\right)\right. \\
& +b_{j}^{+} \beta_{j}^{+}\left(b_{j}^{+}\left\|z_{i j}\right\|+\sum_{i=1}^{n} b_{i j}^{+} M_{i}^{h}+\sum_{i=1}^{n} q_{i j}^{+} M_{i}^{k}+J_{j}^{+}\right) \\
& \left.+\sum_{j=1}^{m} a_{j i}^{+} M_{j}^{f}+\sum_{j=1}^{m} p_{j i}^{+} M_{j}^{g}+I_{i}^{+}+\sum_{i=1}^{n} b_{i j}^{+} M_{i}^{h}+\sum_{i=1}^{n} q_{i j}^{+} M_{i}^{k}+J_{j}^{+}\right] .
\end{aligned}
$$

Then

$$
\begin{aligned}
\left\|z_{i j}(t)\right\| \leq & {\left[1-\frac{\sqrt{2}}{\alpha}\left(\left(a_{i}^{+}\right)^{2} \alpha_{i}^{+}+\left(b_{j}^{+}\right)^{2} \beta_{j}^{+}\right)\right]^{-1}\left\{\sqrt{2}\left(\|\varphi\|^{2}+\|\psi\|^{2}\right)+\frac{\sqrt{2}}{\alpha}\right.} \\
& {\left[a_{i}^{+} \alpha_{i}^{+}\left(\sum_{j=1}^{m} a_{j i}^{+} M_{j}^{f}++\sum_{j=1}^{m} p_{j i}^{+} M_{j}^{g}+I_{i}^{+}\right)\right.} \\
& +b_{j}^{+} \beta_{j}^{+}\left(\sum_{i=1}^{n} b_{i j}^{+} M_{i}^{h}+\sum_{i=1}^{n} q_{i j}^{+} M_{i}^{k}+J_{j}^{+}\right) \\
& \left.\left.+\sum_{j=1}^{m} a_{j i}^{+} M_{j}^{f}+\sum_{j=1}^{m} p_{j i}^{+} M_{j}^{g}+I_{i}^{+}+\sum_{i=1}^{n} b_{i j}^{+} M_{i}^{h}+\sum_{i=1}^{n} q_{i j}^{+} M_{i}^{k}+J_{j}^{+}\right]\right\} .
\end{aligned}
$$

Let

$$
\sigma^{*}=\left[1-\frac{\sqrt{2}}{\alpha}\left(\left(a_{i}^{+}\right)^{2} \alpha_{i}^{+}+\left(b_{j}^{+}\right)^{2} \beta_{j}^{+}\right)\right]^{-1}\left\{\sqrt{2}\left(\|\varphi\|^{2}+\|\psi\|^{2}\right)+\frac{\sqrt{2}}{\alpha}\right.
$$




$$
\begin{aligned}
& {\left[a_{i}^{+} \alpha_{i}^{+}\left(\sum_{j=1}^{m} a_{j i}^{+} M_{j}^{f}++\sum_{j=1}^{m} p_{j i}^{+} M_{j}^{g}+I_{i}^{+}\right)\right.} \\
& +b_{j}^{+} \beta_{j}^{+}\left(\sum_{i=1}^{n} b_{i j}^{+} M_{i}^{h}+\sum_{i=1}^{n} q_{i j}^{+} M_{i}^{k}+J_{j}^{+}\right) \\
& \left.\left.+\sum_{j=1}^{m} a_{j i}^{+} M_{j}^{f}+\sum_{j=1}^{m} p_{j i}^{+} M_{j}^{g}+I_{i}^{+}+\sum_{i=1}^{n} b_{i j}^{+} M_{i}^{h}+\sum_{i=1}^{n} q_{i j}^{+} M_{i}^{k}+J_{j}^{+}\right]\right\} .
\end{aligned}
$$

Then it follows that $\left|x_{i}(t)\right| \leq \sigma^{*},\left|y_{j}(t)\right| \leq \sigma^{*}, i=1,2, \cdots, n, j=1,2, \cdots, m$ for all $t>0$. This completes the proof of Lemma 2.4 .

\section{Main results}

In this section, we present our main result that there exists the exponentially stable anti-periodic solution of (1.1).

Theorem 3.1. Assume that (H1)-(H4) hold true. Then any solution $\left(x^{*}(t), y^{*}(t)\right)^{T}$ of system (1.1) is globally exponentially stable.

Proof. Let $u_{i}(t)=x_{i}(t)-x_{i}^{*}(t), v_{j}(t)=y_{j}(t)-y_{j}^{*}(t), i=1,2, \cdots, n, j=1,2, \cdots, m$. It follows from system (2.4) that

$$
\left\{\begin{aligned}
\frac{d u_{i}(t)}{d t}= & -a_{i}(t) u_{i}(t)+a_{i}(t) \int_{t-\alpha_{i}(t)}^{t}\left\{-a_{i}(s) u_{i}\left(s-\alpha_{i}(s)\right)\right. \\
& +\sum_{j=1}^{m} a_{j i}(s)\left[f_{j}\left(y_{j}\left(s-\tau_{j i}(s)\right)\right)-f_{j}\left(y_{j}^{*}\left(s-\tau_{j i}(s)\right)\right)\right] \\
& \left.+\sum_{j=1}^{m} p_{j i}(t)\left[g_{j}\left(y_{j}^{\prime}\left(s-\sigma_{j i}(s)\right)\right)-g_{j}\left(y_{j}^{\prime *}\left(s-\sigma_{j i}(s)\right)\right)\right]\right\} d s \\
& +\sum_{j=1}^{m} a_{j i}(t)\left[f_{j}\left(y_{j}\left(t-\tau_{j i}(t)\right)\right)-f_{j}\left(y_{j}^{*}\left(t-\tau_{j i}(t)\right)\right)\right] \\
& +\sum_{j=1}^{m} p_{j i}(t)\left[g_{j}\left(y_{j}^{\prime}\left(t-\sigma_{j i}(t)\right)\right)-g_{j}\left(y_{j}^{\prime *}\left(t-\sigma_{j i}(t)\right)\right)\right], \\
\frac{d v_{j}(t)}{d t}= & -b_{j}(t) v_{j}(t)+b_{j}(t) \int_{t-\beta_{j}(t)}^{t}\left\{-b_{j}(s) v_{j}\left(s-\beta_{j}(s)\right)\right. \\
& +\sum_{i=1}^{n} b_{i j}(s)\left[h_{i}\left(x_{i}\left(s-\xi_{i j}(s)\right)\right)-h_{i}\left(x_{i}^{*}\left(s-\xi_{i j}(s)\right)\right)\right] \\
& +\sum_{i=1}^{n} q_{i j}(s)\left[k_{i}\left(x_{i}^{\prime}\left(s-\varsigma_{i j}(s)\right)\right)-k_{i}\left(x_{i}^{\prime *}\left(s-\varsigma_{i j}(s)\right)\right)\right\} d s \\
& +\sum_{i=1}^{n} b_{i j}(t)\left[h_{i}\left(x_{i}\left(t-\xi_{i j}(t)\right)\right)-h_{i}\left(x_{i}^{*}\left(t-\xi_{i j}(t)\right)\right)\right] \\
& +\sum_{i=1}^{n} q_{i j}(t)\left[k_{i}\left(x_{i}^{\prime}\left(t-\varsigma_{i j}(t)\right)\right)-k_{i}\left(x_{i}^{\prime *}\left(t-\varsigma_{i j}(t)\right)\right)\right],
\end{aligned}\right.
$$


which leads to

$$
\left\{\begin{aligned}
\frac{1}{2} \frac{d u_{i}^{2}(t)}{d t}= & -a_{i}(t) u_{i}^{2}(t)+a_{i}(t) u_{i}(t) \int_{t-\alpha_{i}(t)}^{t}\left\{-a_{i}(s) u_{i}\left(s-\alpha_{i}(s)\right)\right. \\
& +\sum_{j=1}^{m} a_{j i}(s)\left[f_{j}\left(y_{j}\left(s-\tau_{j i}(s)\right)\right)-f_{j}\left(y_{j}^{*}\left(s-\tau_{j i}(s)\right)\right)\right] \\
& \left.+\sum_{j=1}^{m} p_{j i}(t)\left[g_{j}\left(y_{j}^{\prime}\left(s-\sigma_{j i}(s)\right)\right)-g_{j}\left(y_{j}^{\prime *}\left(s-\sigma_{j i}(s)\right)\right)\right]\right\} d s \\
& +u_{i}(t) \sum_{j=1}^{m} a_{j i}(t)\left[f_{j}\left(y_{j}\left(t-\tau_{j i}(t)\right)\right)-f_{j}\left(y_{j}^{*}\left(t-\tau_{j i}(t)\right)\right)\right] \\
& +u_{i}(t) \sum_{j=1}^{m} p_{j i}(t)\left[g_{j}\left(y_{j}^{\prime}\left(t-\sigma_{j i}(t)\right)\right)-g_{j}\left(y_{j}^{*}\left(t-\sigma_{j i}(t)\right)\right)\right] \\
\frac{1}{2} \frac{d v_{j}^{2}(t)}{d t}= & -b_{j}(t) v_{j}^{2}(t)+b_{j}(t) v_{j}(t) \int_{t-\beta_{j}(t)}^{t}\left\{-b_{j}(s) v_{j}\left(s-\beta_{j}(s)\right)\right. \\
& +\sum_{i=1}^{n} b_{i j}(s)\left[h_{i}\left(x_{i}\left(s-\xi_{i j}(s)\right)\right)-h_{i}\left(x_{i}^{*}\left(s-\xi_{i j}(s)\right)\right)\right] \\
& +\sum_{i=1}^{n} q_{i j}(s)\left[k_{i}\left(x_{i}^{\prime}\left(s-\varsigma_{i j}(s)\right)\right)-k_{i}\left(x_{i}^{\prime *}\left(s-\varsigma_{i j}(s)\right)\right)\right\} d s \\
& +v_{j}(t) \sum_{i=1}^{n} b_{i j}(t)\left[h_{i}\left(x_{i}\left(t-\xi_{i j}(t)\right)\right)-h_{i}\left(x_{i}^{*}\left(t-\xi_{i j}(t)\right)\right)\right] \\
& +v_{j}(t) \sum_{i=1}^{n} q_{i j}(t)\left[k_{i}\left(x_{i}^{\prime}\left(t-\varsigma_{i j}(t)\right)\right)-k_{i}\left(x_{i}^{*}\left(t-\varsigma_{i j}(t)\right)\right)\right] .
\end{aligned}\right.
$$

Then

$$
\left\{\begin{aligned}
\frac{d u_{i}^{2}(t)}{d t}= & -2 a_{i}(t) u_{i}^{2}(t)+2 a_{i}(t) u_{i}(t) \int_{t-\alpha_{i}(t)}^{t}\left\{-a_{i}(s) u_{i}\left(s-\alpha_{i}(s)\right)\right. \\
& +\sum_{j=1}^{m} a_{j i}(s)\left[f_{j}\left(y_{j}\left(s-\tau_{j i}(s)\right)\right)-f_{j}\left(y_{j}^{*}\left(s-\tau_{j i}(s)\right)\right)\right] \\
& \left.+\sum_{j=1}^{m} p_{j i}(t)\left[g_{j}\left(y_{j}^{\prime}\left(s-\sigma_{j i}(s)\right)\right)-g_{j}\left(y_{j}^{*}\left(s-\sigma_{j i}(s)\right)\right)\right]\right\} d s \\
& +2 u_{i}(t) \sum_{j=1}^{m} a_{j i}(t)\left[f_{j}\left(y_{j}\left(t-\tau_{j i}(t)\right)\right)-f_{j}\left(y_{j}^{*}\left(t-\tau_{j i}(t)\right)\right)\right] \\
& +2 u_{i}(t) \sum_{j=1}^{m} p_{j i}(t)\left[g_{j}\left(y_{j}^{\prime}\left(t-\sigma_{j i}(t)\right)\right)-g_{j}\left(y_{j}^{*}\left(t-\sigma_{j i}(t)\right)\right)\right], \\
\frac{d v_{j}^{2}(t)}{d t}= & -2 b_{j}(t) v_{j}^{2}(t)+2 b_{j}(t) v_{j}(t) \int_{t-\beta_{j}(t)}^{t}\left\{-b_{j}(s) v_{j}\left(s-\beta_{j}(s)\right)\right. \\
& +\sum_{i=1}^{n} b_{i j}(s)\left[h_{i}\left(x_{i}\left(s-\xi_{i j}(s)\right)\right)-h_{i}\left(x_{i}^{*}\left(s-\xi_{i j}(s)\right)\right)\right] \\
& +\sum_{i=1}^{n} q_{i j}(s)\left[k_{i}\left(x_{i}^{\prime}\left(s-\varsigma_{i j}(s)\right)\right)-k_{i}\left(x_{i}^{*}\left(s-\varsigma_{i j}(s)\right)\right)\right\} d s \\
& +2 v_{j}(t) \sum_{i=1}^{n} b_{i j}(t)\left[h_{i}\left(x_{i}\left(t-\xi_{i j}(t)\right)\right)-h_{i}\left(x_{i}^{*}\left(t-\xi_{i j}(t)\right)\right)\right] \\
& +2 v_{j}(t) \sum_{i=1}^{n} q_{i j}(t)\left[k_{i}\left(x_{i}^{\prime}\left(t-\varsigma_{i j}(t)\right)\right)-k_{i}\left(x_{i}^{\prime *}\left(t-\varsigma_{i j}(t)\right)\right)\right] .
\end{aligned}\right.
$$


In view of the condition (H1), we get

$$
\left\{\begin{aligned}
\frac{d u_{i}^{2}(t)}{d t} \leq & -2 a_{i}^{-} u_{i}^{2}(t)+a_{i}^{+} \alpha_{i}^{+}\left[a_{i}^{+}\left(u_{i}^{2}+u_{i}^{2}\left(t-\alpha_{i}(t)\right)\right)\right. \\
& +\sum_{j=1}^{m} a_{j i}^{+}\left(L_{j f}^{2 \xi_{j}} u_{i}^{2}(t)+L_{j f}^{2\left(1-\xi_{j}\right)} v_{j}^{2}\left(t-\tau_{j i}(t)\right)\right) \\
& \left.+\sum_{j=1}^{m} p_{j i}^{+}\left(L_{j g}^{2 \gamma_{j}} u_{i}^{2}(t)+L_{j f}^{2\left(1-\gamma_{j}\right)} v_{j}^{\prime 2}\left(t-\sigma_{j i}(t)\right)\right)\right] \\
& +\sum_{j=1}^{m} a_{j i}^{+}\left[L_{j f}^{2 \xi_{j}} u_{i}^{2}(t)+L_{j f}^{2\left(1-\xi_{j}\right)} v_{j}^{2}\left(t-\tau_{j i}(t)\right)\right] \\
& +\sum_{j=1}^{m} p_{j i}^{+}\left[L_{j g}^{2 \gamma_{j}} u_{i}^{2}(t)+L_{j f}^{2\left(1-\gamma_{j}\right)} v_{j}^{2}\left(t-\sigma_{j i}(t)\right)\right] \\
\frac{d v_{i}^{2}(t)}{d t} \leq & -2 b_{j}^{-} v_{j}^{2}(t)+b_{j}^{+} \beta_{j}^{+}\left[b_{j}^{+}\left(v_{j}^{2}+v_{j}^{2}\left(t-\beta_{j}(t)\right)\right)\right. \\
& +\sum_{i=1}^{n} b_{i j}^{+}\left(L_{i h}^{2 \varepsilon_{i}} v_{j}^{2}(t)+L_{i h}^{2\left(1-\varepsilon_{i}\right)} u_{i}^{2}\left(t-\xi_{i j}(t)\right)\right) \\
& \left.+\sum_{i=1}^{n} q_{i j}^{+}\left(L_{i k}^{2 \iota_{i}} v_{j}^{2}(t)+L_{i h}^{2\left(1-\iota_{i}\right)} u_{i}^{\prime 2}\left(t-\varsigma_{i j}(t)\right)\right)\right] \\
& +\sum_{i=1}^{n} b_{i j}^{+}\left[L_{i h}^{2 \varepsilon_{i}} v_{j}^{2}(t)+L_{i h}^{2\left(1-\varepsilon_{i}\right)} u_{i}^{2}\left(t-\xi_{i j}(t)\right)\right] \\
& +\sum_{i=1}^{n} q_{i j}^{+}\left[L_{i k}^{2 \iota_{i}} v_{j}^{2}(t)+L_{i h}^{2\left(1-\iota_{i}\right)} u_{i}^{\prime 2}\left(t-\varsigma_{i j}(t)\right)\right]
\end{aligned}\right.
$$

where $0 \leq \xi_{j}, \gamma_{j}, \varepsilon_{i}, \iota_{i} \leq 1, i=1,2, \cdots, n, j=1,2, \cdots, m$.

Now we consider the following Lyapunov function

$$
\begin{aligned}
V(t)= & e^{\beta t} \sum_{i=1}^{n} u_{i}^{2}(t)+e^{\beta t} \sum_{j=1}^{m} v_{j}^{2}(t) \\
& +\sum_{i=1}^{n} \sum_{j=1}^{m} a_{i}^{+} \alpha_{i}^{+} a_{j i}^{+} L_{j f}^{2\left(1-\xi_{j}\right)} \int_{t-\tau_{j i}(t)}^{t} e^{\beta\left(s+\tau_{j i}(t)\right)} v_{j}^{2}(s) d s \\
& +\sum_{i=1}^{n} \sum_{j=1}^{m} a_{i}^{+} \alpha_{i}^{+} p_{j i}^{+} L_{j f}^{2\left(1-\gamma_{j}\right)} \int_{t-\sigma_{j i}(t)}^{t} e^{\beta\left(s+\sigma_{j i}(t)\right)} v_{j}^{\prime 2}(s) d s \\
& +\sum_{i=1}^{n} \sum_{j=1}^{m} a_{j i}^{+} L_{j f}^{2\left(1-\xi_{j}\right)} \int_{t-\tau_{j i}(t)}^{t} e^{\beta\left(s+\tau_{j i}(t)\right)} v_{j}^{2}(s) d s \\
& +\sum_{i=1}^{n} \sum_{j=1}^{m} p_{j i}^{+} L_{j f}^{2\left(1-\gamma_{j}\right)} \int_{t-\sigma_{j i}(t)}^{t} e^{\beta\left(s+\sigma_{j i}(t)\right)} v_{j}^{\prime 2}(s) d s \\
& +\sum_{j=1}^{m} \sum_{i=1}^{n} b_{j}^{+} \beta_{j}^{+} b_{i j}^{+} L_{j h}^{2\left(1-\varepsilon_{i}\right)} \int_{t-\xi_{i j}(t)}^{t} e^{\beta\left(s+\xi_{i j}(t)\right)} u_{i}^{2}(s) d s \\
& +\sum_{j=1}^{m} \sum_{i=1}^{n} b_{j}^{+} \beta_{j}^{+} q_{i j}^{+} L_{j h}^{2\left(1-\iota_{i}\right)} \int_{t-\varsigma_{i j}(t)}^{t} e^{\beta\left(s+\varsigma_{i j}(t)\right)} u_{i}^{\prime 2}(s) d s
\end{aligned}
$$




$$
\begin{aligned}
& +\sum_{j=1}^{m} \sum_{i=1}^{n} b_{i j}^{+} L_{j h}^{2\left(1-\varepsilon_{i}\right)} \int_{t-\xi_{i j}(t)}^{t} e^{\beta\left(s+\xi_{i j}(t)\right)} u_{i}^{2}(s) d s \\
& +\sum_{j=1}^{m} \sum_{i=1}^{n} q_{i j}^{+} L_{j h}^{2\left(1-\iota_{i}\right)} \int_{t-\varsigma_{i j}(t)}^{t} e^{\beta\left(s+\varsigma_{i j}(t)\right)} u_{i}^{\prime 2}(s) d s
\end{aligned}
$$

where $\beta$ is given by Lemma 2.3. Differentiating $V(t)$ along solutions to system (1.1), together with (3.3), we have

$$
\begin{aligned}
& \frac{d V(t)}{d t} \leq \beta e^{\beta t}\left[\sum_{i=1}^{n} u_{i}^{2}(t)+\sum_{j=1}^{m} v_{j}^{2}(t)\right] \\
& +e^{\beta t} \sum_{j=1}^{n}\left\{-2 a_{i}^{-} u_{i}^{2}(t)+a_{i}^{+} \alpha_{i}^{+}\left[a_{i}^{+}\left(u_{i}^{2}+u_{i}^{2}\left(t-\alpha_{i}(t)\right)\right)\right.\right. \\
& +\sum_{j=1}^{m} a_{j i}^{+}\left(L_{j f}^{2 \xi_{j}} u_{i}^{2}(t)+L_{j f}^{2\left(1-\xi_{j}\right)} v_{j}^{2}\left(t-\tau_{j i}(t)\right)\right) \\
& \left.+\sum_{j=1}^{m} p_{j i}^{+}\left(L_{j g}^{2 \gamma_{j}} u_{i}^{2}(t)+L_{j f}^{2\left(1-\gamma_{j}\right)} v_{j}^{\prime 2}\left(t-\sigma_{j i}(t)\right)\right)\right] \\
& +\sum_{j=1}^{m} a_{j i}^{+}\left[L_{j f}^{2 \xi_{j}} u_{i}^{2}(t)+L_{j f}^{2\left(1-\xi_{j}\right)} v_{j}^{2}\left(t-\tau_{j i}(t)\right)\right] \\
& \left.+\sum_{j=1}^{m} p_{j i}^{+}\left[L_{j g}^{2 \gamma_{j}} u_{i}^{2}(t)+L_{j f}^{2\left(1-\gamma_{j}\right)} v_{j}^{\prime 2}\left(t-\sigma_{j i}(t)\right)\right]\right\} \\
& +e^{\beta t} \sum_{j=1}^{m}\left\{-2 b_{j}^{-} v_{j}^{2}(t)+b_{j}^{+} \beta_{j}^{+}\left[b_{j}^{+}\left(v_{j}^{2}+v_{j}^{2}\left(t-\beta_{j}(t)\right)\right)\right.\right. \\
& +\sum_{i=1}^{n} b_{i j}^{+}\left(L_{i h}^{2 \varepsilon_{i}} v_{j}^{2}(t)+L_{i h}^{2\left(1-\varepsilon_{i}\right)} u_{i}^{2}\left(t-\xi_{i j}(t)\right)\right) \\
& \left.+\sum_{i=1}^{n} q_{i j}^{+}\left(L_{i k}^{2 \iota_{i}} v_{j}^{2}(t)+L_{i h}^{2\left(1-\iota_{i}\right)} u_{i}^{\prime 2}\left(t-\varsigma_{i j}(t)\right)\right)\right] \\
& +\sum_{i=1}^{n} b_{i j}^{+}\left[L_{i h}^{2 \varepsilon_{i}} v_{j}^{2}(t)+L_{i h}^{2\left(1-\varepsilon_{i}\right)} u_{i}^{2}\left(t-\xi_{i j}(t)\right)\right] \\
& \left.+\sum_{i=1}^{n} q_{i j}^{+}\left[L_{i k}^{2 \iota_{i}} u_{j}^{\prime 2}(t)+L_{i h}^{2\left(1-\iota_{i}\right)} u_{i}^{\prime 2}\left(t-\varsigma_{i j}(t)\right)\right]\right\} \\
& +\sum_{i=1}^{n} \sum_{j=1}^{m} a_{i}^{+} \alpha_{i}^{+} a_{j i}^{+} L_{j f}^{2\left(1-\xi_{j}\right)}\left[e^{\beta\left(t+\tau_{j i}(t)\right)} v_{j}^{2}(t)-e^{\beta t} v_{j}^{2}\left(t-\tau_{j i}(t)\right)\right] \\
& +\sum_{i=1}^{n} \sum_{j=1}^{m} a_{i}^{+} \alpha_{i}^{+} p_{j i}^{+} L_{j f}^{2\left(1-\gamma_{j}\right)}\left[e^{\beta\left(t+\sigma_{j i}(t)\right)} v_{j}^{\prime 2}(t)-e^{\beta t} v_{j}^{\prime 2}\left(t-\sigma_{j i}(t)\right)\right] \\
& +\sum_{i=1}^{n} \sum_{j=1}^{m} a_{j i}^{+} L_{j f}^{2\left(1-\xi_{j}\right)}\left[e^{\beta\left(t+\tau_{j i}(t)\right)} v_{j}^{\prime 2}(t)-e^{\beta t} v_{j}^{\prime 2}\left(t-\tau_{j i}(t)\right)\right] \\
& +\sum_{i=1}^{n} \sum_{j=1}^{m} p_{j i}^{+} L_{j f}^{2\left(1-\gamma_{j}\right)}\left[e^{\beta\left(t+\sigma_{j i}(t)\right)} v_{j}^{\prime 2}(t)-e^{\beta t} v_{j}^{\prime 2}\left(t-\sigma_{j i}(t)\right)\right]
\end{aligned}
$$




$$
\begin{aligned}
& +\sum_{j=1}^{m} \sum_{i=1}^{n} b_{j}^{+} \beta_{j}^{+} b_{i j}^{+} L_{j h}^{2\left(1-\varepsilon_{i}\right)}\left[e^{\beta\left(t+\xi_{i j}(t)\right)} u_{i}^{2}(t)-e^{\beta t} u_{i}^{2}\left(t-\xi_{i j}(t)\right)\right] \\
& +\sum_{j=1}^{m} \sum_{i=1}^{n} b_{j}^{+} \beta_{j}^{+} q_{i j}^{+} L_{j h}^{2\left(1-\iota_{i}\right)}\left[e^{\beta\left(t+\varsigma_{i j}(t)\right)} u_{i}^{\prime 2}(t)-e^{\beta t} u_{i}^{\prime 2}\left(t-\varsigma_{i j}(t)\right)\right] \\
& +\sum_{j=1}^{m} \sum_{i=1}^{n} b_{i j}^{+} L_{j h}^{2\left(1-\varepsilon_{i}\right)}\left[e^{\beta\left(t+\xi_{i j}(t)\right)} u_{i}^{2}(t)-e^{\beta t} u_{i}^{2}\left(t-\xi_{i j}(t)\right)\right] \\
& +\sum_{j=1}^{m} \sum_{i=1}^{n} q_{i j}^{+} L_{j h}^{2\left(1-\iota_{i}\right)}\left[e^{\beta\left(t+\varsigma_{i j}(t)\right)} u_{i}^{\prime 2}(t)-e^{\beta t} u_{i}^{\prime 2}\left(t-\varsigma_{i j}(t)\right)\right] \\
& =e^{\beta t} \sum_{i=1}^{n}\left[\beta-2 a_{i}^{-}+a_{i}^{+} \alpha_{i}^{+}\left(a_{i}^{+}+\sum_{j=1}^{m} a_{j i}^{+} L_{j f}^{2 \xi_{j}}+\sum_{j=1}^{m} p_{j i}^{+} L_{j g}^{2 \gamma_{j}}\right)\right. \\
& \left.+\sum_{j=1}^{m} a_{j i}^{+} L_{j f}^{2 \xi_{j}}+\sum_{j=1}^{m} p_{j i}^{+} L_{j g}^{2 \gamma_{j}}+\sum_{j=1}^{m} b_{j}^{+} \beta_{j} b_{i j}^{+} L_{j h}^{2\left(1-\varepsilon_{i}\right)} e^{\beta \tau}+\sum_{j=1}^{m} b_{i j}^{+} L_{j h}^{2\left(1-\varepsilon_{i}\right)} e^{\beta \tau}\right] u_{i}^{2}(t) \\
& +e^{\beta t} \sum_{i=1}^{n}\left[\sum_{j=1}^{m} q_{i j}^{+} L_{j h}^{2 \iota_{i}} e^{\beta \tau}+\sum_{j=1}^{m} b_{j}^{+} \beta_{j}^{+} q_{i j}^{+} L_{j h}^{2\left(1-\iota_{i}\right)} e^{\beta \tau}\right] u_{i}^{\prime 2}(t) \\
& +e^{\beta t} \sum_{j=1}^{m}\left[\beta-2 b_{j}^{-}+b_{j}^{+} \beta_{j}^{+}\left(b_{j}^{+}+\sum_{i=1}^{n} b_{i j}^{+} L_{i h}^{2 \varepsilon_{i}}+\sum_{i=1}^{n} q_{i j}^{+} L_{i h}^{2 \iota_{i}}\right)\right. \\
& \left.+\sum_{i=1}^{n} b_{i j}^{+} L_{i h}^{2 \varepsilon_{i}}+\sum_{i=1}^{n} a_{i}^{+} \alpha_{i}^{+} a_{j i}^{+} L_{j f}^{2\left(1-\varepsilon_{j}\right)} e^{\beta \tau}+\sum_{i=1}^{n} a_{j i}^{+} L_{j f}^{2\left(1-\xi_{j}\right)} e^{\beta \tau}\right] v_{j}^{2}(t) \\
& +e^{\beta t} \sum_{j=1}^{m}\left[\sum_{i=1}^{n} a_{i}^{+} \alpha_{i}^{+} p_{j i}^{+} L_{j g}^{2\left(1-\gamma_{j}\right)} e^{\beta \tau}+\sum_{i=1}^{n} p_{j i}^{+} L_{j g}^{2\left(1-\gamma_{j}\right)} e^{\beta \tau}+\sum_{i=1}^{n} a_{j i}^{+} L_{j f}^{2\left(1-\xi_{j}\right)} e^{\beta \tau}\right] v_{j}^{\prime 2}(t) \\
& \leq e^{\beta t} \sum_{i=1}^{n}\left[\beta-2 a_{i}^{-}+a_{i}^{+} \alpha_{i}^{+}\left(a_{i}^{+}+\sum_{j=1}^{m} a_{j i}^{+} L_{j f}^{2 \xi_{j}}+\sum_{j=1}^{m} p_{j i}^{+} L_{j g}^{2 \gamma_{j}}\right)\right. \\
& +\sum_{j=1}^{m} a_{j i}^{+} L_{j f}^{2 \xi_{j}}+\sum_{j=1}^{m} p_{j i}^{+} L_{j g}^{2 \gamma_{j}}+\sum_{j=1}^{m} b_{j}^{+} \beta_{j} b_{i j}^{+} L_{j h}^{2\left(1-\varepsilon_{i}\right)} e^{\beta \tau}+\sum_{j=1}^{m} b_{i j}^{+} L_{j h}^{2\left(1-\varepsilon_{i}\right)} e^{\beta \tau} \\
& \left.+\sum_{j=1}^{m} q_{i j}^{+} L_{j h}^{2 \iota_{i}} e^{\beta \tau}+\sum_{j=1}^{m} b_{j}^{+} \beta_{j}^{+} q_{i j}^{+} L_{j h}^{2\left(1-\iota_{i}\right)} e^{\beta \tau}\right]\left|\tilde{u}_{i}(t)\right|^{2} \\
& +e^{\beta t} \sum_{i=1}^{n}\left[\beta-2 b_{j}^{-}+b_{j}^{+} \beta_{j}^{+}\left(b_{j}^{+}+\sum_{i=1}^{n} b_{i j}^{+} L_{i h}^{2 \varepsilon_{i}}+\sum_{i=1}^{n} q_{i j}^{+} L_{i h}^{2 \iota_{i}}\right)\right. \\
& +\sum_{i=1}^{n} b_{i j}^{+} L_{i h}^{2 \varepsilon_{i}}+\sum_{i=1}^{n} a_{i}^{+} \alpha_{i}^{+} a_{j i}^{+} L_{j f}^{2\left(1-\varepsilon_{j}\right)} e^{\beta \tau}+\sum_{i=1}^{n} a_{j i}^{+} L_{j f}^{2\left(1-\xi_{j}\right)} e^{\beta \tau} \\
& \left.+\sum_{i=1}^{n} a_{i}^{+} \alpha_{i}^{+} p_{j i}^{+} L_{j g}^{2\left(1-\gamma_{j}\right)} e^{\beta \tau}+\sum_{i=1}^{n} p_{j i}^{+} L_{j g}^{2\left(1-\gamma_{j}\right)} e^{\beta \tau},+\sum_{i=1}^{n} a_{j i}^{+} L_{j f}^{2\left(1-\xi_{j}\right)} e^{\beta \tau}\right]\left|\tilde{v}_{j}(t)\right|^{2} .
\end{aligned}
$$

where

$$
\left|\tilde{u}_{i}(t)\right|=\max _{t \in R}\left\{\left|u_{i}(t)\right|,\left|u_{i}^{\prime}(t)\right|\right\},\left|\tilde{v}_{j}(t)\right|=\max _{t \in R}\left\{\left|v_{j}(t)\right|,\left|v_{j}^{\prime}(t)\right|\right\} .
$$


It follows from Lemma 2.3 that $\frac{d V(t)}{d t} \leq 0$, which implies that $V(t) \leq V(0)$ for all $t>0$. Thus

$$
\begin{aligned}
& e^{\beta t}\left[\sum_{i=1}^{n} u_{i}^{2}(t)+\sum_{j=1}^{m} v_{j}^{2}(t)\right] \leq \sum_{i=1}^{n} u_{i}^{2}(0)+\sum_{j=1}^{m} v_{i}^{2}(0) \\
& +\sum_{i=1}^{n} \sum_{j=1}^{m} a_{i}^{+} \alpha_{i}^{+} a_{j i}^{+} L_{j f}^{2\left(1-\xi_{j}\right)} \int_{-\tau_{j i}(0)}^{0} e^{\beta\left(s+\tau_{j i}(0)\right)} v_{j}^{2}(s) d s \\
& +\sum_{i=1}^{n} \sum_{j=1}^{m} a_{i}^{+} \alpha_{i}^{+} p_{j i}^{+} L_{j f}^{2\left(1-\gamma_{j}\right)} \int_{-\sigma_{j i}(0)}^{t} e^{\beta\left(s+\sigma_{j i}(0)\right)} v_{j}^{\prime 2}(s) d s \\
& +\sum_{i=1}^{n} \sum_{j=1}^{m} a_{j i}^{+} L_{j f}^{2\left(1-\xi_{j}\right)} \int_{-\tau_{j i}(0)}^{0} e^{\beta\left(s+\tau_{j i}(0)\right)} v_{j}^{2}(s) d s \\
& +\sum_{i=1}^{n} \sum_{j=1}^{m} p_{j i}^{+} L_{j f}^{2\left(1-\gamma_{j}\right)} \int_{-\sigma_{j i}(0)}^{0} e^{\beta\left(s+\sigma_{j i}(0)\right)} v_{j}^{\prime 2}(s) d s \\
& +\sum_{j=1}^{m} \sum_{i=1}^{n} b_{j}^{+} \beta_{j}^{+} b_{i j}^{+} L_{j h}^{2\left(1-\varepsilon_{i}\right)} \int_{-\xi_{i j}(0)}^{0} e^{\beta\left(s+\xi_{i j}(0)\right)} u_{i}^{2}(s) d s \\
& +\sum_{j=1}^{m} \sum_{i=1}^{n} b_{j}^{+} \beta_{j}^{+} q_{i j}^{+} L_{j h}^{2\left(1-\iota_{i}\right)} \int_{-\varsigma_{i j}(0)}^{0} e^{\beta\left(s+\varsigma_{i j}(0)\right)} u_{i}^{\prime 2}(s) d s \\
& +\sum_{j=1}^{m} \sum_{i=1}^{n} b_{i j}^{+} L_{j h}^{2\left(1-\varepsilon_{i}\right)} \int_{-\xi_{i j}(0)}^{0} e^{\beta\left(s+\xi_{i j}(0)\right)} u_{i}^{2}(s) d s \\
& +\sum_{j=1}^{m} \sum_{i=1}^{n} q_{i j}^{+} L_{j h}^{2\left(1-\iota_{i}\right)} \int_{-\varsigma_{i j}(0)}^{0} e^{\beta\left(s+\varsigma_{i j}(0)\right)} u_{i}^{\prime 2}(s) d s \\
& \leq\left\|\varphi-\varphi^{*}\right\|^{2}+\left\|\psi-\psi^{*}\right\|^{2} \\
& +\sum_{i=1}^{n} \sum_{j=1}^{m} a_{i}^{+} \alpha_{i}^{+} a_{j i}^{+} L_{j f}^{2\left(1-\xi_{j}\right)} \frac{1}{\beta} e^{\beta \tau}\left\|\psi-\psi^{*}\right\|^{2} \\
& +\sum_{i=1}^{n} \sum_{j=1}^{m} a_{i}^{+} \alpha_{i}^{+} p_{j i}^{+} L_{j f}^{2\left(1-\gamma_{j}\right)} \frac{1}{\beta} e^{\beta \tau}\left\|\psi-\psi^{*}\right\|^{2} \\
& +\sum_{i=1}^{n} \sum_{j=1}^{m} a_{j i}^{+} L_{j f}^{2\left(1-\xi_{j}\right)} \frac{1}{\beta} e^{\beta \tau}\left\|\psi-\psi^{*}\right\|^{2} \\
& +\sum_{i=1}^{n} \sum_{j=1}^{m} p_{j i}^{+} L_{j f}^{2\left(1-\gamma_{j}\right)} \frac{1}{\beta} e^{\beta \tau}\left\|\psi-\psi^{*}\right\|^{2} \\
& +\sum_{j=1}^{m} \sum_{i=1}^{n} b_{j}^{+} \beta_{j}^{+} b_{i j}^{+} L_{j h}^{2\left(1-\varepsilon_{i}\right)} \frac{1}{\beta} e^{\beta \tau}\left\|\varphi-\varphi^{*}\right\|^{2} \\
& +\sum_{j=1}^{m} \sum_{i=1}^{n} b_{j}^{+} \beta_{j}^{+} q_{i j}^{+} L_{j h}^{2\left(1-\iota_{i}\right)} \frac{1}{\beta} e^{\beta \tau}\left\|\varphi-\varphi^{*}\right\|^{2} \\
& +\sum_{j=1}^{m} \sum_{i=1}^{n} b_{i j}^{+} L_{j h}^{2\left(1-\varepsilon_{i}\right)} \frac{1}{\beta} e^{\beta \tau}\left\|\varphi-\varphi^{*}\right\|^{2} \\
& +\sum_{j=1}^{m} \sum_{i=1}^{n} q_{i j}^{+} L_{j h}^{2\left(1-\iota_{i}\right)} \frac{1}{\beta} e^{\beta \tau}\left\|\varphi-\varphi^{*}\right\|^{2}
\end{aligned}
$$




$$
\begin{aligned}
= & {\left[1+\sum_{j=1}^{m} \sum_{i=1}^{n} b_{j}^{+} \beta_{j}^{+} b_{i j}^{+} L_{j h}^{2\left(1-\varepsilon_{i}\right)} \frac{1}{\beta} e^{\beta \tau}+\sum_{j=1}^{m} \sum_{i=1}^{n} b_{j}^{+} \beta_{j}^{+} q_{i j}^{+} L_{j h}^{2\left(1-\iota_{i}\right)} \frac{1}{\beta} e^{\beta \tau}\right.} \\
& \left.+\sum_{j=1}^{m} \sum_{i=1}^{n} b_{i j}^{+} L_{j h}^{2\left(1-\varepsilon_{i}\right)} \frac{1}{\beta} e^{\beta \tau}+\sum_{j=1}^{m} \sum_{i=1}^{n} q_{i j}^{+} L_{j h}^{2\left(1-\iota_{i}\right)} \frac{1}{\beta} e^{\beta \tau}\right]\left\|\varphi-\varphi^{*}\right\|^{2} \\
& +\left[1+\sum_{i=1}^{n} \sum_{j=1}^{m} a_{i}^{+} \alpha_{i}^{+} a_{j i}^{+} L_{j f}^{2\left(1-\xi_{j}\right)} \frac{1}{\beta} e^{\beta \tau}+\sum_{i=1}^{n} \sum_{j=1}^{m} a_{i}^{+} \alpha_{i}^{+} p_{j i}^{+} L_{j f}^{2\left(1-\gamma_{j}\right)} \frac{1}{\beta} e^{\beta \tau}\right. \\
& \left.+\sum_{i=1}^{n} \sum_{j=1}^{m} a_{j i}^{+} L_{j f}^{2\left(1-\xi_{j}\right)} \frac{1}{\beta} e^{\beta \tau}+\sum_{i=1}^{n} \sum_{j=1}^{m} p_{j i}^{+} L_{j f}^{2\left(1-\gamma_{j}\right)} \frac{1}{\beta} e^{\beta \tau}\right]\left\|\psi-\psi^{*}\right\|^{2} .
\end{aligned}
$$

Let

$$
\begin{aligned}
\mu_{1}= & +\sum_{j=1}^{m} \sum_{i=1}^{n} b_{j}^{+} \beta_{j}^{+} b_{i j}^{+} L_{j h}^{2\left(1-\varepsilon_{i}\right)} \frac{1}{\beta} e^{\beta \tau}+\sum_{j=1}^{m} \sum_{i=1}^{n} b_{j}^{+} \beta_{j}^{+} q_{i j}^{+} L_{j h}^{2\left(1-\iota_{i}\right)} \frac{1}{\beta} e^{\beta \tau} \\
& +\sum_{j=1}^{m} \sum_{i=1}^{n} b_{i j}^{+} L_{j h}^{2\left(1-\varepsilon_{i}\right)} \frac{1}{\beta} e^{\beta \tau}+\sum_{j=1}^{m} \sum_{i=1}^{n} q_{i j}^{+} L_{j h}^{2\left(1-\iota_{i}\right)} \frac{1}{\beta} e^{\beta \tau}, \\
\mu_{2}=1 & +\sum_{i=1}^{n} \sum_{j=1}^{m} a_{i}^{+} \alpha_{i}^{+} a_{j i}^{+} L_{j f}^{2\left(1-\xi_{j}\right)} \frac{1}{\beta} e^{\beta \tau}+\sum_{i=1}^{n} \sum_{i=1}^{m} a_{i}^{+} \alpha_{i}^{+} p_{j i}^{+} L_{j f}^{2\left(1-\gamma_{j}\right)} \frac{1}{\beta} e^{\beta \tau} \\
& +\sum_{i=1}^{n} \sum_{j=1}^{m} a_{j i}^{+} L_{j f}^{2\left(1-\xi_{j}\right)} \frac{1}{\beta} e^{\beta \tau}+\sum_{i=1}^{n} \sum_{j=1}^{m} p_{j i}^{+} L_{j f}^{2\left(1-\gamma_{j}\right)} \frac{1}{\beta} e^{\beta \tau}
\end{aligned}
$$

and choose

$$
M=\max \left\{\mu_{1}, \mu_{2}\right\}>1
$$

Then Eq.3.7) can be rewritten as

$$
\sum_{i=1}^{n} u_{i}^{2}(t)+\sum_{i=1}^{m} v_{j}^{2}(t) \leq M e^{-\beta t}\left[\left\|\varphi-\varphi^{*}\right\|^{2}+\left\|\psi-\psi^{*}\right\|^{2}\right]
$$

for all $t>0$. Then

$$
\sum_{i=1}^{n}\left|x_{i}(t)-x_{i}^{*}(t)\right|^{2}+\sum_{i=1}^{m}\left|y_{j}(t)-y_{j}^{*}(t)\right|^{2} \leq M e^{-\beta t}\left[\left\|\varphi-\varphi^{*}\right\|^{2}+\left\|\psi-\psi^{*}\right\|^{2}\right]
$$

for all $t>0$. Thus the solution $(x(t), y(t))^{T}$ of system $(1.1)$ is globally exponentially stable.

Theorem 3.2. If (H1)-(H4) are fulfilled, then system (1.1) has exactly one T-anti-periodic solution which is globally stable.

Proof. It follows from system (1.1) and (H2) that for each $k \in N$, we have

$$
\begin{aligned}
\frac{d}{d t}\left[(-1)^{k+1} x_{i}(t+(k+1) T)\right]= & (-1)^{k+1}\left[-a_{i}(t+(k+1) T) x_{i}\left(t+(k+1) T-\alpha_{i}(t+(k+1) T)\right)\right. \\
& +\sum_{j=1}^{m} a_{j i}(t+(k+1) T) f_{j}\left(y_{j}\left(t+(k+1) T-\tau_{j i}(t+(k+1) T)\right)\right) \\
& +\sum_{j=1}^{m} p_{j i}(t+(k+1) T) g_{j}\left(y_{j}^{\prime}\left(t+(k+1) T-\sigma_{j i}(t+(k+1) T)\right)\right)
\end{aligned}
$$




$$
\begin{aligned}
& \left.+I_{i}(t+(k+1) T)\right] \\
= & -a_{i}(t)(-1)^{k+1} x_{i}\left(t+(k+1) T-\alpha_{i}(t)\right) \\
& +\sum_{j=1}^{m} a_{j i}(t) f_{j}\left((-1)^{k+1} y_{j}\left(t+(k+1) T-\tau_{j i}(t)\right)\right) \\
& +\sum_{j=1}^{m} p_{j i}(t) g_{j}\left((-1)^{k+1} y_{j}^{\prime}\left(t+(k+1) T-\sigma_{j i}(t)\right)\right)+I_{i}(t), \\
\frac{d}{d t}\left[(-1)^{k+1} y_{j}(t+(k+1) T)\right]= & -1)^{k+1}\left[-b_{j}(t+(k+1) T) y_{j}\left(t+(k+1) T-\beta_{j}(t+(k+1) T)\right)\right. \\
& +\sum_{i=1}^{n} b_{i j}(t+(k+1) T) h_{i}\left(x_{i}\left(t+(k+1) T-\xi_{i j}(t+(k+1) T)\right)\right) \\
& +\sum_{i=1}^{n} q_{i j}(t+(k+1) T) k_{i}\left(x_{i}^{\prime}\left(t+(k+1) T-\varsigma_{i j}(t+(k+1) T)\right)\right) \\
& \left.+J_{i}(t+(k+1) T)\right] \\
= & -b_{j}(t)(-1)^{k+1} y_{j}\left(t+(k+1) T-\beta_{j}(t)\right) \\
& +\sum_{i=1}^{n} b_{i j}(t) h_{i}\left((-1)^{k+1} x_{i}\left(t+(k+1) T-\xi_{i j}(t)\right)\right) \\
& +\sum_{i=1}^{n} q_{i j}(t) k_{i}\left((-1)^{k+1} x_{i}^{\prime}\left(t+(k+1) T-\varsigma_{i j}(t)\right)\right)+J_{i}(t) .
\end{aligned}
$$

Let

$$
\begin{aligned}
& \bar{x}(t)=\left((-1)^{k+1} x_{1}(t+(k+1) T),(-1)^{k+1} x_{2}(t+(k+1) T), \cdots,(-1)^{k+1} x_{n}(t+(k+1) T)\right)^{T}, \\
& \bar{y}(t)=\left((-1)^{k+1} y_{1}(t+(k+1) T),(-1)^{k+1} y_{2}(t+(k+1) T), \cdots,(-1)^{k+1} y_{m}(t+(k+1) T)\right)^{T} .
\end{aligned}
$$

Obviously, for any $k \in N,(\bar{x}(t), \bar{y}(t))$ is also the solution of system (1.1). If the initial functions $\varphi_{i}(s)$, $\psi_{j}(s)(i=1,2, \cdots, n, j=1,2, \cdots, m)$ are bounded, it follows from Theorem 3.1 that there exists a constant $\gamma>1$ such that

$$
\begin{aligned}
\left|(-1)^{k+1} x_{i}(t+(k+1) T)-(-1)^{k} x_{i}(t+k T)\right| & \leq M e^{-\beta(t+k T)} \sup _{-\tau \leq s \leq 0} \sum_{i=1}^{n}\left|x_{i}(t+T)+x_{i}(s)\right|^{2} \\
& \leq \gamma e^{-\beta(t+k T)},
\end{aligned}
$$

where $t+k T>0, i=1,2, \cdots, n$. Since for any $k \in N$ we have

$$
(-1)^{k+1} x_{i}(t+(k+1) T)=x_{i}(t)+\sum_{j=0}^{k}\left[(-1)^{j+1} x_{i}(t+(j+1) T)-(-1)^{j} x_{i}(t+j T)\right] .
$$

Then

$$
(-1)^{k+1} x_{i}(t+(k+1) T) \leq\left|x_{i}(t)\right|+\sum_{j=0}^{k}\left|(-1)^{j+1} x_{i}(t+(j+1) T)-(-1)^{j} x_{i}(t+j T)\right| .
$$

By Lemma 2.4, we know that the solutions of system (1.1) are bounded. In view of (3.10) and (3.12), we can easily see that $\left\{(-1)^{k+1} x_{i}(t+(k+1) T)\right\}$ uniformly converges to a continuous function $x^{*}(t)=$ $\left(x_{1}^{*}(t), x_{2}^{*}(t), \cdots, x_{n}^{*}(t)\right)^{T}$ on any compact set of $R^{n}$. In a similar way, we can easily prove that $\left\{(-1)^{k+1} y_{j}(t+\right.$ 
$(k+1) T)\}$ uniformly converges to a continuous function $y^{*}(t)=\left(y_{1}^{*}(t), y_{2}^{*}(t), \cdots, y_{m}^{*}(t)\right)^{T}$ on any compact set of $R^{m}$.

Now we show that $x^{*}(t)$ is $T$-anti-periodic solution of (1.1). Firstly, $x^{*}(t)$ is $T$-anti-periodic, since

$$
\begin{aligned}
x^{*}(t+T) & =\lim _{k \rightarrow \infty}(-1)^{k} x(t+T+k T) \\
& =-\lim _{(k+1) \rightarrow \infty}(-1)^{k+1} x(t+(k+1) T)=-x^{*}(t) .
\end{aligned}
$$

Then we can conclude that $x_{i}^{*}(t)$ is $T$-anti-periodic on $R$. Similarly, $y_{j}^{*}(t)$ is also $T$-anti-periodic on $R$. Thus we can conclude that $\left(x^{*}(t), y^{*}(t)\right)^{T}$ is the solution of system (1.1).

In fact, together with the continuity of the right side of system (1.1), let $k \rightarrow \infty$, we can easily get

$$
\left\{\begin{aligned}
\frac{d x_{i}^{*}(t)}{d t}= & -a_{i}(t) x_{i}^{*}\left(t-\alpha_{i}(t)\right)+\sum_{j=1}^{m} a_{j i}(t) f_{j}\left(y_{j}^{*}\left(t-\tau_{j i}(t)\right)\right) \\
& +\sum_{j=1}^{m} p_{j i}(t) g_{j}\left(y_{j}^{\prime *}\left(t-\sigma_{j i}(t)\right)\right)+I_{i}(t) \\
\frac{d y_{j}^{*}(t)}{d t}= & -b_{j}(t) y_{j}^{*}\left(t-\beta_{j}(t)\right)+\sum_{i=1}^{n} b_{i j}(t) h_{i}\left(x_{i}^{*}\left(t-\xi_{i j}(t)\right)\right) \\
& +\sum_{i=1}^{n} q_{i j}(t) k_{i}\left(x_{i}^{\prime *}\left(t-\varsigma_{i j}(t)\right)\right)+J_{i}(t) .
\end{aligned}\right.
$$

Therefore, $\left(x^{*}(t), y^{*}(t)\right)^{T}$ is a solution of 1.1 . Finally, by applying Theorem 3.1. it is easy to check that $\left(x^{*}(t), y^{*}(t)\right)^{T}$ is globally exponentially stable. This completes the proof of Theorem 3.2 .

\section{An example}

In this section, we give an example to illustrate our main results derived in previous sections. Consider the following neutral BAM neural network with time-varying delays in the leakage terms

$$
\left\{\begin{aligned}
\frac{d x_{1}(t)}{d t}= & -a_{1}(t) x_{1}\left(t-\alpha_{1}(t)\right)+\sum_{j=1}^{2} a_{j 1}(t) f_{j}\left(y_{j}\left(t-\tau_{j 1}(t)\right)\right) \\
& +\sum_{j=1}^{2} p_{j 1}(t) g_{j}\left(y_{j}^{\prime}\left(t-\sigma_{j 1}(t)\right)\right)+I_{1}(t), \\
\frac{d x_{2}(t)}{d t}= & -a_{2}(t) x_{2}\left(t-\alpha_{2}(t)\right)+\sum_{j=1}^{2} a_{j 2}(t) f_{j}\left(y_{j}\left(t-\tau_{j 2}(t)\right)\right) \\
& +\sum_{j=1}^{2} p_{j 2}(t) g_{j}\left(y_{j}^{\prime}\left(t-\sigma_{j 2}(t)\right)\right)+I_{2}(t), \\
\frac{d y_{1}(t)}{d t}= & -b_{1}(t) y_{1}\left(t-\beta_{1}(t)\right)+\sum_{i=1}^{2} b_{i 1}(t) h_{i}\left(x_{i}\left(t-\xi_{i 1}(t)\right)\right) \\
& +\sum_{i=1}^{n} q_{i 1}(t) k_{i}\left(x_{i}^{\prime}\left(t-\varsigma_{i 1}(t)\right)\right)+J_{1}(t), \\
\frac{d y_{2}(t)}{d t}= & -b_{2}(t) y_{2}\left(t-\beta_{2}(t)\right)+\sum_{i=1}^{2} b_{i 2}(t) h_{i}\left(x_{i}\left(t-\xi_{i 2}(t)\right)\right) \\
& +\sum_{i=1}^{2} q_{i 2}(t) k_{i}\left(x_{i}^{\prime}\left(t-\varsigma_{i 2}(t)\right)\right)+J_{2}(t),
\end{aligned}\right.
$$


where

$$
\begin{aligned}
& {\left[\begin{array}{ll}
\alpha_{1}(t) & \alpha_{2}(t) \\
\beta_{1}(t) & \beta_{2}(t)
\end{array}\right]=\left[\begin{array}{ll}
0.04|\sin t| & 0.04|\sin t| \\
0.03|\cos t| & 0.03|\cos t|
\end{array}\right]} \\
& {\left[\begin{array}{ll}
a_{11}(t) & a_{12}(t) \\
a_{21}(t) & a_{22}(t)
\end{array}\right]=\left[\begin{array}{ll}
0.2|\sin t| & 0.2|\sin t| \\
0.3|\cos t| & 0.3|\cos t|
\end{array}\right]} \\
& {\left[\begin{array}{ll}
p_{11}(t) & p_{12}(t) \\
p_{21}(t) & p_{22}(t)
\end{array}\right]=\left[\begin{array}{ll}
0.02|\sin t| & 0.02|\sin t| \\
0.03|\cos t| & 0.03|\cos t|
\end{array}\right]} \\
& {\left[\begin{array}{ll}
b_{11}(t) & b_{12}(t) \\
b_{21}(t) & b_{22}(t)
\end{array}\right]=\left[\begin{array}{ll}
0.2|\sin t| & 0.2|\sin t| \\
0.3|\cos t| & 0.3|\cos t|
\end{array}\right]} \\
& {\left[\begin{array}{ll}
q_{11}(t) & q_{12}(t) \\
q_{21}(t) & q_{22}(t)
\end{array}\right]=\left[\begin{array}{ll}
0.02|\sin t| & 0.02|\sin t| \\
0.03|\cos t| & 0.03|\cos t|
\end{array}\right],} \\
& {\left[\begin{array}{ll}
I_{1}(t) & I_{2}(t) \\
J_{1}(t) & J_{2}(t)
\end{array}\right]=\left[\begin{array}{ll}
0.5 \cos t & 0.5 \cos t \\
0.5 \sin t & 0.5 \sin t
\end{array}\right],\left[\begin{array}{ll}
a_{1} & a_{2} \\
b_{1} & b_{2}
\end{array}\right]=\left[\begin{array}{ll}
2 & 2 \\
2 & 2
\end{array}\right] .}
\end{aligned}
$$

Set $f_{j}(u)=g_{j}(u)=h_{i}(u)=k_{i}(u)=\frac{1}{2}(|u+1|-|u-1|), i, j=1,2$. Then $L_{j f}=g_{j g}=L_{i h}=g_{i k}=M_{j}^{f}=$ $M_{j}^{g}=M_{i}^{h}=M_{i}^{k}=1, \alpha_{1}^{+}=\alpha_{1}^{+}=0.04, \beta_{1}^{+}=\beta_{2}^{+}=0.03, a_{11}^{+}=a_{12}^{+}=0.2, a_{21}^{+}=a_{22}^{+}=0.3, b_{11}^{+}=b_{12}^{+}=$ $0.2, b_{21}^{+}=b_{22}^{+}=0.3, p_{11}^{+}=p_{12}^{+}=0.02, p_{21}^{+}=p_{22}^{+}=0.03, q_{11}^{+}=q_{12}^{+}=0.02, q_{21}^{+}=q_{22}^{+}=0.03, \tau=0.03$. It is easy to verify that

$$
\frac{\sqrt{2}}{\alpha}\left(\left(a_{i}^{+}\right)^{2} \alpha_{i}^{+}+\left(b_{j}^{+}\right)^{2} \beta_{j}^{+}\right)=\frac{\sqrt{2}}{2}(4 \times 0.04+4 \times 0.03)=0.12726<1
$$

and

$$
\begin{aligned}
& -2 a_{1}^{-}+a_{1}^{+} \alpha_{1}^{+}\left(a_{1}^{+}+\sum_{j=1}^{2} a_{j 1}^{+} L_{j f}^{2 \xi_{j}}+\sum_{j=1}^{2} p_{j 1}^{+} L_{j g}^{2 \gamma_{j}}\right) \\
& +\sum_{j=1}^{2} a_{j 1}^{+} L_{j f}^{2 \xi_{j}}+\sum_{j=1}^{2} p_{j 1}^{+} L_{j g}^{2 \gamma_{j}}+\sum_{j=1}^{2} b_{j}^{+} \beta_{j} b_{1 j}^{+} L_{j h}^{2\left(1-\varepsilon_{1}\right)} \\
& +\sum_{j=1}^{2} b_{1 j}^{+} L_{j h}^{2\left(1-\varepsilon_{1}\right)}+\sum_{j=1}^{2} q_{1 j}^{+} L_{j h}^{2 \iota_{1}}+\sum_{j=1}^{2} b_{j}^{+} \beta_{j}^{+} q_{1 j}^{+} L_{j h}^{2\left(1-\iota_{1}\right)} \\
= & -2 \times 2+2 \times 0.04 \times(2+0.5+0.06)+0.5+0.05+2 \times 0.03 \\
& \times 0.2 \times 2+0.4+0.04+2 \times 0.03 \times 0.02 \times 2=-2.7796<0, \\
& -2 a_{2}^{-}+a_{2}^{+} \alpha_{2}^{+}\left(a_{2}^{+}+\sum_{j=1}^{2} a_{j 2}^{+} L_{j f}^{2 \xi_{j}}+\sum_{j=1}^{2} p_{j 2}^{+} L_{j g}^{2 \gamma_{j}}\right) \\
& +\sum_{j=1}^{2} a_{j 2}^{+} L_{j f}^{2 \xi_{j}}+\sum_{j=1}^{2} p_{j 2}^{+} L_{j g}^{2 \gamma_{j}}+\sum_{j=1}^{2} b_{j}^{+} \beta_{j} b_{2 j}^{+} L_{j h}^{2\left(1-\varepsilon_{2}\right)} \\
& +\sum_{j=1}^{2} b_{2 j}^{+} L_{j h}^{2\left(1-\varepsilon_{2}\right)}+\sum_{j=1}^{2} q_{2 j}^{+} L_{j h}^{2 \iota_{2}}+\sum_{j=1}^{2} b_{j}^{+} \beta_{j}^{+} q_{i j}^{+} L_{j h}^{2\left(1-\iota_{2}\right)}=-2.04<0, \\
= & -2 \times 2+2 \times 0.04 \times(2+0.5+0.06)+0.5+0.05+2 \times 0.03 \\
& \times 0.3 \times 2+0.6+0.06+2 \times 0.03 \times 0.03 \times 2=-2.5788<0 \\
& -2 b_{1}^{-}+b_{1}^{+} \beta_{1}^{+}\left(b_{1}^{+}+\sum_{i=1}^{2} b_{i 1}^{+} L_{i h}^{2 \varepsilon_{i}}+\sum_{i=1}^{2} q_{i 1}^{+} L_{i h}^{2 \iota_{i}}\right)
\end{aligned}
$$




$$
\begin{aligned}
& +\sum_{i=1}^{2} b_{i 1}^{+} L_{i h}^{2 \varepsilon_{i}}+\sum_{i=1}^{2} a_{i}^{+} \alpha_{i}^{+} a_{1 i}^{+} L_{1 f}^{2\left(1-\varepsilon_{1}\right)}+\sum_{i=1}^{2} a_{1 i}^{+} L_{1 f}^{2\left(1-\xi_{1}\right)} \\
& +\sum_{i=1}^{2} a_{i}^{+} \alpha_{i}^{+} p_{1 i}^{+} L_{1 g}^{2\left(1-\gamma_{1}\right)}+\sum_{i=1}^{2} p_{1 i}^{+} L_{1 g}^{2\left(1-\gamma_{1}\right)}+\sum_{i=1}^{2} a_{1 i}^{+} L_{1 f}^{2\left(1-\xi_{1}\right)} \\
= & -2 \times 2+2 \times 0.03 \times(2+0.5+0.05)+0.5+0.05+2 \times 0.04 \\
& \times 0.3+0.6+2 \times 0.04 \times 0.02 \times 2+0.04+0.4=-2.2798<0 \\
& -2 b_{2}^{-}+b_{2}^{+} \beta_{2}^{+}\left(b_{2}^{+}+\sum_{i=1}^{2} b_{i 2}^{+} L_{i h}^{2 \varepsilon_{i}}+\sum_{i=1}^{2} q_{i 2}^{+} L_{i h}^{2 \iota_{i}}\right) \\
& +\sum_{i=1}^{2} b_{i 2}^{+} L_{i h}^{2 \varepsilon_{i}}+\sum_{i=1}^{2} a_{i}^{+} \alpha_{i}^{+} a_{2 i}^{+} L_{2 f}^{2\left(1-\varepsilon_{2}\right)}+\sum_{i=1}^{2} a_{2 i}^{+} L_{2 f}^{2\left(1-\xi_{2}\right)} \\
& +\sum_{i=1}^{2} a_{i}^{+} \alpha_{i}^{+} p_{2 i}^{+} L_{2 g}^{2\left(1-\gamma_{2}\right)}+\sum_{i=1}^{2} p_{2 i}^{+} L_{2 g}^{2\left(1-\gamma_{2}\right)}+\sum_{i=1}^{2} a_{2 i}^{+} L_{2 f}^{2\left(1-\xi_{2}\right)} \\
= & -2 \times 2+2 \times 0.03 \times(2+0.5+0.05)+0.5+0.05+2 \times 0.04 \\
& \times 0.3+0.6+2 \times 0.04 \times 0.03 \times 2+0.06+0.6=-2.0582<0 .
\end{aligned}
$$

Then all the conditions (H1)-(H4) hold. Thus system (4.1) has exactly one $\pi$-anti-periodic solution which is globally exponentially stable.

\section{Conclusions}

In this paper, we investigated the asymptotical behavior of a neutral BAM neural networks with timevarying delays in the leakage terms. Applying the fundamental solution matrix of coefficient matrix, we derive a series of new sufficient conditions to guarantee the existence and global exponential stability of an anti-periodic solution for the neutral BAM neural networks with time-varying delays in the leakage terms. The obtained conditions are easily to check in practice. Finally, an example is given to illustrative the feasibility and effectiveness.

\section{Acknowledgments}

The authors are grateful to supporting by National Natural Science Foundation of China (No.11261010), Natural Science and Technology Foundation of Guizhou Province (J[2015]2025) and 125 Special Major Science and Technology of Department of Education of Guizhou Province ([2012]011).

\section{References}

[1] M. S. Ali, P. Balasubramaniam, Global exponential stability of uncertain fuzzy BAM neural networks with timevarying delays, Chaos Solitons Fractals, 42 (2009), 2191-2199.1.

[2] A. P. Chen, L. H. Huang, J. D. Cao, Existence and stability of almost periodic solution for BAM neural networks with delays, Appl. Math. Comput., 137 (2003), 177-193.1

[3] Q. Y. Fan, W. T. Wang, X. J. Yi, Anti-periodic solutions for a class of nonlinear nth-order differential equations with delays, J. Comput. Appl. Math., 230 (2009), 762-769.1

[4] S. H. Gong, Anti-periodic solutions for a class of Cohen-Grossberg neural networks, Comput. Math. Appl., 58 (2009), 341-347.1

[5] K. Gopalsamy, Stability and Oscillations in Delay Differential Equations of Populations Dynamics, Kluwer Academic Publishers, Dordrecht, (1992).1.

[6] K. Gopalsamy, Leakage delays in BAM, J. Math. Anal. Appl., 325 (2007), 1117-1132.1

[7] S. J. Guo, L. H. Huang, Hopf bifurcating periodic orbits in a ring of neurons with delays, Phys. D, 183 (2003), 19-44.1. 
[8] S. Haykin, Neural Networks, Prentice-Hall, NJ, (1994).1.

[9] D. W. C. Ho, J. L. Liang, J. Lam, Global exponential stability of impulsive high-order BAM neural networks with time-varying delays, Neural Netw., 19 (2006), 1581-1590.1

[10] Z. D. Huang, L. Q. Peng, M. Xu, Anti-periodic solutions for high-order cellular neural networks with time-varying delays, Electron J. Differ. Equ., 2010 (2010), 9 pages.1

[11] H. J. Jiang, Z. D. Teng, Boundedness, periodic solutions and global stability for cellular neural networks with variable coefficients and infinite delays, Neurocomputing, 72 (2009), 2455-2463.1]

[12] B. Kosok, Neural Networks and Fuzzy Systems, Prentice-Hall, NewDelhi, (1992).1

[13] S. Lakshmanan, J. H. Park, T. H. Lee, H. Y. Jung, R. Rakkiyappan, Stability criteria for BAM neural networks with leakage delays and probabilistic time-varying delays, Appl. Math. Comput., 219 (2013), 9408-9423. 1

[14] Y. K. Li, Anti-periodic solutions to impulsive shunting inhibitory cellular neural networks with distributed delays on time scales, Commun. Nonlinear Sci. Numer. Simul., 16 (2011), 3326-3336.1

[15] Y. K. Li, X. R. Chen, L. Zhao, Stability and existence of periodic solutions to delayed Cohen-Grossberg BAM neural networks with impulses on time scales, Neurocomputing, 72 (2009), 1621-1673.1]

[16] Y. K. Li, Y. Q. Li, Existence and exponential stability of almost periodic solution for neutral delay BAM neural networks with time-varying delays in leakage terms, J. Frankin Inst., 350 (2013), 2808-2825.11, 1

[17] Y. K. Li, C. Wang, Existence and global exponential stability of equilibrium for discrete-time fuzzy BAM neural networks with variable delays and impulses, Fuzzy Sets Syst., 217 (2013), 62-79.1.

[18] Y. K. Li, E. L. Xu, T. W. Zhang, Existence and stability of anti-periodic solution for a class of generalized neural networks with impulsives and arbitrary delays on time scales, J. Inequal. Appl., 2010 (2010), 19 pages.1.

[19] Y. K. Li, L. Yang, Anti-periodic solutions for Cohen-Grossberg neural netowrks with bounded and unbounded dealys, Commun. Nonlinear Sci. Numer. Simul., 14 (2009), 3134-3140.1

[20] Y. K. Li, L. Yang, W. Q. Wu, Anti-periodic solutions for a class of Cohen-Grossberg neural networks with time-varying on time scales, Int. J. Syst. Sci., 42 (2011), 1127-1132.1

[21] Y. G. Liu, Z. X. Huang, L. P. Chen, Almost periodic solution of impulsive Hopfield neural networks with finite distributed delays, Neural Comput. Appl., 21 (2012), 821-831.1

[22] D. Y. Liu, W. J. Wu, H. T. Liu, J. W. Zhang, Anti-periodic solutions for interval general Bidirectional Associative Memory (BAM) neural networks with impulses on time scales, J. Infor. Comput. Sci., 8 (2011), 3847-3857.1.

[23] C. X. Ou, Anti-periodic solutions for high-order Hopfield neural networks, Comput. Math. Appl., 56 (2008), 1838-1844.1

[24] L. J. Pan, J. D. Cao, Anti-periodic solution for delayed cellular neural networks with impulsive effects, Nonlinear Anal. Real World Appl., 12 (2011), 3014-3027.1

[25] J. H. Park, S. M. Lee, O. M. Kwon, On exponential stability of bidirectional associative memory neural networks with time-varying delays, Chaos Solitons Fractals, 39 (2009), 1083-1091.1.

[26] G. Q. Peng, L. H. Huang, Anti-periodic solutions for shunting inhibitory cellular neural networks with continuously distributed delays, Nonlinear Anal. Real World Appl., 10 (2009), 2434-2440.1

[27] L. Peng, W. T. Wang, Anti-periodic solutions for shunting inhibitory cellular neural networks with time-varying delays in leakage terms, Neurocomputing, 111 (2013), 27-33.1.

[28] R. Raja, S. M. Anthoni, Global exponential stability of BAM neural networks with time-varying delays: The discrete-time case, Commun. Nonlinear Sci. Numer. Simul., 16 (2011), 613-622.1.

[29] R. Sakthivel, A. Arunkumar, K. Mathiyalagan, S. M. Anthoni, Robust passivity analysis of fuzzy Cohen-Grossberg BAM neural networks with time-varying delays, Appl. Math. Comput., 218 (2011), 3799-3809.1

[30] R. Samidurai, R. Sakthivel, S. M. Anthoni, Global asymptotic stability of BAM neural networks mixed delays and impulses, Appl. Math. Comput., 212 (2009), 113-119.1

[31] J. Y. Shao, Anti-periodic solutions for shunting inhibitory cellular neural networks with time-varying delays, Phys. Lett. A, 372 (2008), 5011-5016.1

[32] P. L. Shi, L. Z. Dong, Existence and exponential stability of anti-periodic solutions of Hopfield neural networks with impulses, Appl. Math. Comput., 216 (2010), 623-630.1

[33] Y. Y. Song, Y. Y. Han, Y. H. Peng, Stability and Hopf bifurcation in an unidirectional ring of $n$ neurons with distributed delays, Neurocomputing, 121 (2013), 442-452.1

[34] Q. K. Song, Z. D. Wang, An analysis on existence and global exponential stability of periodic solutions for BAM neural networks with time-varying delays, Nonlinear Anal. Real World Appl., 8 (2007), 1224-1234.1.

[35] X. R. Wei, Z. P. Qiu, Anti-periodic solutions for BAM neural networks with time delays, Appl. Math. Comput., 221 (2013), 221-229.1

[36] A. P. Zhang, Existence and exponential stability of anti-periodic solutions for HCNNs with time-varying leakage delays, Adv. Difference. Equ., 2013 (2013), 14 pages.1]

[37] Z. Q. Zhang, K. Y. Liu, Existence and global exponential stability of a periodic solution to interval general bidirectional associative memory (BAM) neural networks with multiple delays on time scales, Neural Netw., 24 (2011), 427-439.1

[38] Z. Q. Zhang, K. Y. Liu, Y. Yang, New LMI-based condition on global asymptotic stability concerning BAM neural networks of neutral type, Neurocomputing, 81 (2012), 24-32.1

[39] Z. Q. Zhang, W. B. Liu, D. M. Zhou, Global asymptotic stability to a generalized Cohen-Grossberg BAM neural 
networks of neutral type delays, Neural Netw., 25 (2012), 94-105.1.

[40] Z. Q. Zhang, Y. Yang, Y. S. Huang, Global exponential stability of interval general BAM neural networks with reaction-diffusion terms and multiple time-varying delays, Neural Netw., 24 (2011), 457-465.1

[41] C. R. Zhang, B. D. Zheng, L. C. Wang, Multiple Hopf bifurcations of symmetric BAM neural network model with delay, Appl. Math. Lett., 22 (2009), 616-622.1.

[42] Z. Q. Zhang, D. M. Zhou, Existence and global exponential stability of a periodic solution for a discrete-time interval general BAM neural networks, J. Franklin Inst., 347 (2010), 763-780.1 